\title{
Multiplicity Results for the $p(x)$-Laplacian Equation with Singular Nonlinearities and Nonlinear Neumann Boundary Condition
}

\author{
K. Saoudi, M. Kratou, and S. Alsadhan \\ College of Sciences at Dammam, University of Dammam, Dammam 31441, Saudi Arabia \\ Correspondence should be addressed to K. Saoudi; kasaoudi@gmail.com
}

Received 5 April 2016; Accepted 22 June 2016

Academic Editor: Julio D. Rossi

Copyright (C) 2016 K. Saoudi et al. This is an open access article distributed under the Creative Commons Attribution License, which permits unrestricted use, distribution, and reproduction in any medium, provided the original work is properly cited.

We investigate the singular Neumann problem involving the $p(x)$-Laplace operator: $\left(P_{\lambda}\right)\left\{-\Delta_{p(x)} u+|u|^{p(x)-2} u=1 / u^{\delta(x)}+f(x, u)\right.$, in $\Omega ; u>0$, in $\Omega ;|\nabla u|^{p(x)-2} \partial u / \partial v=\lambda u^{q(x)}$, on $\partial \Omega$, where $\Omega \subset \mathbb{R}^{N}(N \geq 2)$ is a bounded domain with $C^{2}$ boundary, $\lambda$ is a positive parameter, and $p(x), q(x), \delta(x)$, and $f(x, u)$ are assumed to satisfy assumptions (H0)-(H5) in the Introduction. Using some variational techniques, we show the existence of a number $\Lambda \in(0, \infty)$ such that problem $\left(P_{\lambda}\right)$ has two solutions for $\lambda \in(0, \Lambda)$, one solution for $\lambda=\Lambda$, and no solutions for $\lambda>\Lambda$.

\section{Introduction}

The purpose of this paper is to study the existence of multiple solutions for the following inhomogeneous singular Neumann problem involving the $p(x)$-Laplace operator:

$$
\left(P_{\lambda}\right) \begin{cases}-\Delta_{p(x)} u+|u|^{p(x)-2} u=\frac{1}{u^{\delta(x)}}+f(x, u), & \text { in } \Omega, \\ u>0, & \text { in } \Omega, \\ |\nabla u|^{p(x)-2} \frac{\partial u}{\partial \nu}=\lambda u^{q(x)}, & \text { on } \partial \Omega .\end{cases}
$$

Here $\Omega \subset \mathbb{R}^{N}(N \geq 2)$ is a bounded domain with $C^{2}$ boundary; $\lambda$ is a positive parameter. For any continuous and bounded function $a$ we define $a^{+}:=\sup a(x)$ and $a^{-}:=$ $\inf a(x)$. Associated with problem $\left(P_{\lambda}\right)$ we have the singular functional $E_{\lambda}: W^{1, p(x)}(\Omega) \rightarrow \mathbb{R}$ given by

$$
\begin{aligned}
E_{\lambda}(u) \stackrel{\operatorname{def}}{=} & \int_{\Omega} \frac{|\nabla u|^{p(x)}}{p(x)} d x+\int_{\Omega} \frac{|u|^{p(x)}}{p(x)} d x \\
& -\int_{\Omega} \frac{|u|^{1-\delta(x)}}{1-\delta(x)} d x-\int_{\Omega} F(x, u) d x \\
& -\lambda \int_{\partial \Omega} \frac{|u|^{q(x)+1}}{q(x)+1} d x,
\end{aligned}
$$

where $F(x, t)=\int_{0}^{t} f(x, s) d s$.
Definition 1. $u \in W^{1, p(x)}(\Omega)$ is called a generalized solution of the equation

$$
-\Delta_{p(x)} u+|u|^{p(x)-2} u=\frac{1}{u^{\delta(x)}}+f(x, u)
$$

if for all $\varphi \in C_{0}^{\infty}(\Omega)$

$$
\begin{gathered}
\int_{\Omega}|\nabla u|^{p(x)-2} \nabla u \nabla \varphi d x+\int_{\Omega}|u|^{p(x)-2} u \varphi d x \\
=\int_{\Omega}|u|^{-\delta(x)} \varphi d x+\int_{\Omega} f(x, u) \varphi d x \\
+\lambda \int_{\partial \Omega}|u|^{q(x)} \varphi d x .
\end{gathered}
$$

Obviously, every weak solution of problem $\left(P_{\lambda}\right)$ is also a generalized solution of (3).

The operator $\Delta_{p(x)} u:=\operatorname{div}\left(|\nabla u|^{p(x)-2} \nabla u\right)$ is called $p(x)$ Laplace where $p$ is a continuous nonconstant function. This differential operator is a natural generalization of the $p$ Laplace operator $\Delta_{p} u:=\operatorname{div}\left(|\nabla u|^{p-2} \nabla u\right)$, where $p>$ 1 is a real constant. However, the $p(x)$-Laplace operator possesses more complicated nonlinearity than $p$-Laplace operator, due to the fact that $\Delta_{p(x)}$ is not homogeneous. This fact implies some difficulties; for example, we cannot use 
the Lagrange Multiplier Theorem in many problems involving this operator.

The study of differential and partial differential equations involving variable exponent is a new and an interesting topic. The interest in studying such problems was stimulated by their applications in elastic mechanics, fluid dynamics, electrorheological fluids, image processing, flow in porous media, calculus of variations, nonlinear elasticity theory, heterogeneous porous media models (see Acerbi and Mingione [1], Diening [2]), and so forth. These physical problems were facilitated by the development of Lebesgue and Sobolev spaces with variable exponent.

At this point, we briefly recall related existence and multiplicity results for elliptic equations with Neumann boundary conditions. Neumann type problems are studied in [3-6] and references therein. The multiplicity result for Neumann problem with Sobolev critical nonlinearity has been studied in [5] where authors considered the problem

$$
\begin{aligned}
-\Delta u+u & =u^{p}, \quad u>0 \text { in } \Omega, \\
\frac{\partial u}{\partial \nu} & =\lambda \psi u^{q} \quad \text { on } \partial \Omega .
\end{aligned}
$$

Here $\Omega \subset \mathbb{R}^{N}, N \geq 3$, and $0<q<1<p \leq 2 N /(N-2)$. They proved the existence of $\widetilde{\Lambda}$ such that problem (5) admits at least two solutions for all $\lambda \in(0, \widetilde{\Lambda})$, one solution when $\lambda=\widetilde{\Lambda}$, and no solutions for $\lambda>\widetilde{\Lambda}$. The problem in two dimensions has been considered in [6] where the authors extended the results obtained by [5].

Results for $p(x)$-Laplacian problems Neumann boundary conditions are rare (see $[7,8])$. In [7], Fan and Deng studied the Neumann problems with $p(x)$-Laplace operator and the nonlinear potential $f(x, u)$ under appropriate assumptions. By using the subsupersolution method and variation method, the authors get the multiplicity of positive solutions. In [8], Sreenadh and Tiwari extend previous works on nonlinear parametric problems with the $p(x)$-Laplace operator to the case where the Neumann boundary condition is nonlinear. Precisely, under supplementary hypotheses on $p, q$, the authors show that there exists a finite number $\Lambda$ such that the posed problem has two solutions for $\lambda \in(0, \Lambda)$, one solution for $\lambda=\Lambda$, and no solutions for $\lambda>\Lambda$.

Before stating our main results, we make the following assumptions throughout this paper:

(H0) $p(\cdot) \in C(\bar{\Omega}), 1<p^{-} \leq p^{+}<N$, and $p^{+} \leq p_{*}^{-}$. As usual, $p^{*}(x)=N p(x) /(N-p(x))$.

(H1) $0<q(x) \in C^{\beta}(\partial \Omega)$ for some $\beta \in(0,1)$ satisfying $0 \leq q^{+}+1<p^{-}\left(p^{-} / p^{+}\right)$.

(H2) $0<\delta(x) \in C(\bar{\Omega})$ satisfying $0<\delta^{-} \leq \delta^{+}<1$.

Let $f: \Omega \times \mathbb{R} \rightarrow \mathbb{R}$ be a nondecreasing Carathéodory function satisfying the following:

(H3) $f(x, 0)=0$ and $f(x, t) t \geq 0$ for all $(x, t) \in \Omega \times \mathbb{R}$.

(H4) There exist $C_{1}, C_{2}>0$ such that, for $\gamma>0, a(x) \in$ $C(\bar{\Omega})$ with $p^{+}-1<\gamma$ and $p^{+}<a^{-}$.

$$
C_{1} u^{\gamma} \leq f(x, t) \leq C_{2} t^{a(x)-1}, \quad \forall x \in \Omega, t>0 .
$$

(H5) There exist a constant $M>0$ and $\theta>p^{+}$such that

$$
0<\theta F(x, t) \leq f(x, t) t, \quad \forall x \in \Omega,|t|>M .
$$

Next we describe in a more precise way our main results.

Theorem 2. Assume that (H0)-(H5) hold and $a(x) \leq$ $p^{*}(x)=N p(x) /(N-p(x))$ in (6). Then there exists $\Lambda>0$ with the following properties:

(1) Problem $\left(P_{\lambda}\right)$ has a solution $u_{\lambda}$ for every $\lambda \in(0, \Lambda)$.

(2) Problem $\left(P_{\lambda}\right)$ has a solution if $\lambda=\Lambda$.

(3) Problem $\left(P_{\lambda}\right)$ does not have any solution if $\lambda>\Lambda$.

Theorem 3. Assume that (H0)-(H5) hold and $a(x)<p^{*}(x)$ in (6). Then, problem $\left(P_{\lambda}\right)$ has at least two distinct solutions $u_{\lambda}(x)$ and $v_{\lambda}(x)$ for every $\lambda \in(0, \Lambda)$.

This paper is organized as follows. In Section 2, we will recall some basic facts about the variable exponent Lebesgue and Sobolev spaces which we will use later. Proofs of our results will be presented in Sections 3 and 5 .

\section{Generalized Lebesgue-Sobolev Spaces Setting}

To deal with the $p(x)$-Laplacian problem, we need to introduce some functional spaces $L^{p(\cdot)}(\Omega), W^{1, p(\cdot)}(\Omega), W_{0}^{1, p(\cdot)}(\Omega)$ and properties of the $p(x)$-Laplacian which we will use later. Denote by $S(\Omega)$ the set of all measurable real-valued functions defined in $\Omega$. Note that two measurable functions are considered as the same element of $S(\Omega)$ when they are equal almost everywhere. Let

$$
L^{p(\cdot)}(\Omega)=\left\{u \in S(\Omega): \int_{\Omega}|u(x)|^{p(x)} d x<\infty\right\},
$$

with the norm

$$
\begin{aligned}
|u|_{p(\cdot)} & =|u|_{L^{p(\cdot)}(\Omega)} \\
& =\inf \left\{\lambda>0: \int_{\Omega}\left|\frac{u(x)}{\lambda}\right|^{p(x)} d x \leq 1\right\} .
\end{aligned}
$$

The space $\left(L^{p(\cdot)}(\Omega),|\cdot|_{p(\cdot)}\right)$ becomes a Banach space. We call it variable exponent Lebesgue space. Moreover, this space is a separable, reflexive, and uniform convex Banach space; see [9, Theorems 1.6, 1.10, and 1.14].

The variable exponent Sobolev space

$$
W^{1, p(\cdot)}(\Omega)=\left\{u \in L^{p^{(\cdot)}}(\Omega):|\nabla u| \in L^{p^{(\cdot)}}(\Omega)\right\}
$$

can be equipped with the norm

$$
\|u\|=|u|_{p(\cdot)}+|\nabla u|_{p(\cdot)}, \quad \forall u \in W^{1, p(\cdot)}(\Omega) .
$$

Note that $W_{0}^{1, p(\cdot)}(\Omega)$ is the closure of $C_{0}^{\infty}(\Omega)$ in $W^{1, p(\cdot)}(\Omega)$ under the norm $\|u\|=|\nabla u|_{p(\cdot)}$. The spaces $W^{1, p(\cdot)}(\Omega)$ and 
$W_{0}^{1, p(\cdot)}(\Omega)$ are separable, reflexive, and uniform convex Banach spaces (see [9, Theorem 2.1]). The inclusion between Lebesgue spaces also generalizes naturally: if $0<|\Omega|<\infty$ and $p_{1}, p_{2}$ are variable exponents so that $p_{1}(x) \leq p_{2}(x)$ almost everywhere in $\Omega$ then there exists the continuous embedding $L^{p_{2}(x)}(\Omega) \hookrightarrow L^{p_{1}(x)}(\Omega)$.

We denote by $L^{q(x)}(\Omega)$ the conjugate space of $L^{p(x)}(\Omega)$, where $1 / q(x)+1 / p(x)=1$. For $u \in L^{p(x)}(\Omega)$ and $v \in L^{q(x)}(\Omega)$, the Hölder type inequality

$$
\left|\int_{\Omega} u(x) v(x) d x\right| \leq\left(\frac{1}{p^{-}}+\frac{1}{q^{-}}\right)|u|_{p(x)}|v|_{q(x)}
$$

holds true.

An important role in manipulating the generalized Lebesgue spaces is played by the modular of the $L^{p(x)}(\Omega)$ space, which is the mapping $\rho_{p(x)}: L^{p(x)}(\Omega) \rightarrow \mathbb{R}$ defined by

$$
\rho_{p(x)}(u)=\int_{\Omega}|u|^{p(x)} d x .
$$

If $\left(u_{n}\right), u \in L^{p(x)}(\Omega)$ and $p^{+}<\infty$, then the following relations hold true.

Lemma 4. Consider the following:

$$
\begin{gathered}
\|u\|_{L^{p(x)}}>1 \Longrightarrow \\
\|u\|_{L^{p(x)}}^{p^{-}} \leq \rho_{p(x)}(u) \leq\|u\|_{L^{p(x)}}^{p^{+}}, \\
\|u\|_{L^{p(x)}}<1 \Longrightarrow \\
\|u\|_{L^{p(x)}}^{p^{+}} \leq \rho_{p(x)}(u) \leq\|u\|_{L^{p(x)}}^{p^{-}}, \\
\left\|u_{n}-u\right\|_{L^{p(x)}} \longrightarrow 0 \quad \text { iff } \rho_{p(x)}\left(u_{n}-u\right) \longrightarrow 0 .
\end{gathered}
$$

The following result generalizes the well-known Sobolev embedding theorem.

Theorem 5 (see $[10,11]$ ). Let $\Omega \subset \mathbb{R}^{N}$ be an open bounded domain with Lipschitz boundary and assume that $p \in C(\bar{\Omega})$ with $p(x)>1$ for each $x \in \bar{\Omega}$. If $r \in C(\bar{\Omega})$ and $p(x) \leq$ $r(x) \leq p^{*}(x)$ for all $x \in \bar{\Omega}$, then there exists a continuous embedding $W^{1, p(x)}(\Omega) \hookrightarrow L^{r(x)}(\Omega)$. Also, the embedding is compact $r(x)<p^{*}(x)$ almost everywhere in $\bar{\Omega}$, where

$$
p^{*}(x)= \begin{cases}\frac{N p(x)}{N-p(x)}, & \text { if } p(x)<N, \\ +\infty, & \text { if } p(x) \geq N .\end{cases}
$$

Now, we recall the following boundary trace embedding theorem from [12].

Theorem 6. Let $\Omega \subset \mathbb{R}^{N}$ be an open bounded domain with Lipschitz boundary. If $q \in C(\partial \Omega)$ such that

$$
1 \leq q(x) \leq \frac{(N-1) p(x)}{N-p(x)}, \quad \forall x \in \partial \Omega,
$$

then $W^{1, p(x)} \hookrightarrow L^{q(x)}(\partial \Omega)$.

Next we give a comparison principle as follows.
Lemma 7 (see $\left[8\right.$, Lemma 3.2]). Let $u, v \in W^{1, p(\cdot)}(\Omega)$ be nonnegative functions satisfying

$$
\begin{aligned}
-\Delta_{p(x)} u+u^{p(x)-1} & \geq-\Delta_{p(x)} v+v^{p(x)-1}, \quad(x \in \Omega), \\
|\nabla u|^{p(x)-2} \frac{\partial u}{\partial \nu} & \geq|\nabla v|^{p(x)-2} \frac{\partial v}{\partial \nu} .
\end{aligned}
$$

Then $u \geq v$ almost everywhere in $\Omega$. [13].

We recall the following strong maximum principle from

Theorem 8. Let $u, v \in C^{1, \beta}(\bar{\Omega})$, for some $0<\beta<1$, satisfy $0 ફ u, 0 ફ v$ and

$$
-\Delta_{p(x)} u-\frac{\lambda}{u^{\delta x}}=g(x) \geq h(x)=-\Delta_{p(x)} v-\frac{\lambda}{v^{\delta(x)}},
$$

with $u=v=0$ on $\partial \Omega$, where $g, h \in L^{\infty}(\Omega)$ are such that $0 \leq g<h$ pointwise everywhere in $\Omega$. If

$$
\begin{aligned}
& \frac{\partial u}{\partial \mathbf{n}}>0, \\
& \frac{\partial v}{\partial \mathbf{n}}>0,
\end{aligned}
$$

on $\partial \Omega$,

where $\mathbf{n}$ is the inward unit normal on $\partial \Omega$, then, the following strong comparison principle holds:

$$
u>v
$$

in $\Omega$;

$$
\text { there is a positive } \epsilon \text { such that } \frac{\partial(u-v)}{\partial \mathbf{n}} \geq \epsilon \text {, }
$$

on $\partial \Omega$.

\section{Existence of a Solution}

In this section, we show the existence of a local minimum for $E_{\lambda}$ in a small neighborhood of the origin in $W^{1, p(x)}(\Omega)$. Firstly, let us define

$$
\Lambda \stackrel{\text { def }}{=} \sup \left\{\lambda>0:\left(P_{\lambda}\right) \text { has a weak solution }\right\} .
$$

Lemma 9. There exists $\lambda_{0}>0$ such that $\left(P_{\lambda}\right)$ admits a solution for $\lambda<\lambda_{0}$.

Proof. Using (14) and the embeddings in Theorem 5, we estimate $E_{\lambda}(u)$ for $\|u\|_{W^{1, p(x)}(\Omega)}=r$ as follows:

$$
\begin{aligned}
E_{\lambda}(u)= & \int_{\Omega} \frac{|\nabla u|^{p(x)}}{p(x)} d x+\int_{\Omega} \frac{|u|^{p(x)}}{p(x)} d x \\
& -\int_{\Omega} \frac{|u|^{1-\delta(x)}}{1-\delta(x)} d x-\int_{\Omega} F(x, u) d x \\
& -\lambda \int_{\partial \Omega} \frac{|u|^{q(x)+1}}{q(x+1)} d x
\end{aligned}
$$




$$
\begin{aligned}
\geq & \|\nabla u\|_{L^{p(x)}(\Omega)}^{p^{+}}+\|u\|_{L^{p(x)}(\Omega)}^{p^{+}}-\|u\|_{L^{p(x)}(\Omega)}^{1-\delta^{+}} \\
& -\|u\|_{L^{p(x)}(\Omega)}^{a^{-}}-\lambda\|u\|_{L^{p(x)}(\partial \Omega)}^{q^{-}+1} \\
\geq & \frac{1}{2^{p^{+}-1}}\left(\|\nabla u\|_{L^{p(x)}(\Omega)}+\|u\|_{L^{p(x)}(\Omega)}\right) p^{p^{+}} \\
& -\|u\|_{W^{1, p(x)}(\Omega)}^{1-\delta^{+}}-\|u\|_{W^{1, p(x)}(\Omega)}^{a^{-}}-\lambda\|u\|_{W^{1, p(x)}(\Omega)}^{q^{-}+1} \\
= & \frac{1}{2^{p^{+}-1}}\|u\|_{W^{1, p(x)}(\Omega)}^{p^{+}}-\|u\|_{W^{1, p(x)}(\Omega)}^{1-\delta^{+}} \\
& -\|u\|_{L^{p(x)}(\Omega)}^{a^{-}}-\lambda\|u\|_{W^{1, p(x)}(\Omega)}^{q^{-}+1} .
\end{aligned}
$$

Hence, noting that $0<\delta^{+}<1$ and $1-\delta^{+}<p^{+}<a^{-}$, we can choose $r_{0}>0$ small enough, and there exists $\delta_{0}>0$ such that

$$
\begin{gathered}
\int_{\Omega} \frac{|\nabla u|^{p(x)}}{p(x)} d x+\int_{\Omega} \frac{|u|^{p(x)}}{p(x)} d x-\int_{\Omega} F(x, u) d x \\
-\lambda \int_{\partial \Omega} \frac{|u|^{q(x)+1}}{q(x+1)} d x \geq 0, \quad \forall u \in B_{r_{0}}, \\
\int_{\Omega} \frac{|\nabla u|^{p(x)}}{p(x)} d x+\int \frac{|u|^{p(x)}}{p(x)} d x-\int_{\Omega} F(x, u) d x \\
-\lambda \int_{\partial \Omega} \frac{|u|^{q(x)+1}}{q(x+1)} d x \geq \delta_{0}, \quad \forall u \in \partial B_{r_{0}} .
\end{gathered}
$$

Moreover, since $W^{1, p(x)}(\Omega) \hookrightarrow L^{1-\delta(x)}(\Omega)$, we have, for $\lambda>0$, small enough,

$$
E_{\lambda} \backslash_{\partial B_{r_{0}}} \geq \frac{\delta_{0}}{2}>0 .
$$

Set

$$
c_{0}=\inf _{u \in B_{r_{0}}} E_{\lambda}(u)
$$

Now, note that, for $t \rightarrow 0^{+}$and $u \in W^{1, p(x)}(\Omega)$, we have

$$
\begin{aligned}
E_{\lambda}(t u)= & \int_{\Omega} \frac{t^{p(x)}|\nabla u|^{p(x)}}{p(x)} d x+\int_{\Omega} \frac{|t u|^{p(x)}}{p(x)} d x \\
& -\int_{\Omega} \frac{|t u|^{1-\delta(x)}}{1-\delta(x)} d x-\int_{\Omega} F(x, t u) d x \\
& -\lambda \int_{\partial \Omega} \frac{|t u|^{q(x)+1}}{q(x)+1} d x \\
\leq & \frac{t^{p^{-}}}{p^{+}} \int_{\Omega}|\nabla u|^{p(x)} d x+\frac{t^{p^{-}}}{p^{+}} \int_{\Omega}|u|^{p(x)} d x \\
& -\frac{t^{1-\delta^{+}}}{1-\delta^{+}} \int_{\Omega}|u|^{1-\delta(x)} d x
\end{aligned}
$$

$$
\begin{aligned}
& -\int_{t u \geq t_{*}} F(x, t u) d x \\
& -\frac{\lambda|t|^{q^{-}+1}}{q^{-}+1} \int_{\partial \Omega}|u|^{q(x)+1} d x \\
\leq & \frac{t^{p^{-}}}{p^{+}} \int_{\Omega}|\nabla u|^{p(x)} d x+\frac{t^{p^{-}}}{p^{+}} \int_{\Omega}|u|^{p(x)} d x \\
& -\frac{t^{1-\delta^{+}}}{1-\delta^{+}} \int_{\Omega}|u|^{1-\delta(x)} d x \\
& -\frac{t^{\theta}}{t_{*}^{\theta}} \int_{t u \geq t_{*}} F(x, t u) d x \\
& -\frac{\lambda|t|^{q^{-}+1}}{q^{-}+1} \int_{\partial \Omega}|u|^{q(x)+1} d x \\
& -\frac{t^{\theta} \int_{u \geq t_{*}}^{p^{-}}}{p^{+}} \int_{\Omega}|\nabla u|^{p(x)} d x+\frac{t^{p^{-}}}{p^{+}} \int_{\Omega}|u|^{p(x)} d x \\
& -\frac{t^{1-\delta^{+}}}{1-\delta^{+}} \int_{\Omega}|u|^{1-\delta(x)} d x \\
q^{-}+1 & |u|^{q(x)+1} d x \\
& \\
& \\
& \\
&
\end{aligned}
$$

Since $1-\delta^{+}<p^{+}<\theta$, this implies that $E_{\lambda}(t u) \rightarrow-\infty$ as $t \rightarrow \infty$. Thus, $\inf _{\|u\|_{W^{1}, p(x)(\Omega) \leq r}} E_{\lambda}(u)=c_{0}<0$. Now, let $\left\{u_{j}\right\} \subset$ $B_{r_{0}}$ be a minimizing sequence for $c_{0}$. Then $\left\|u_{j}\right\|_{W^{1, p(x)}(\Omega)}<r_{0}$ for $r_{0} \in(0, r)$. Now by the Ekeland variational principle, there exists a sequence $\left\{v_{j}\right\}$ such that $\left\|v_{j}\right\|_{W^{1, p(x)}(\Omega)}<r$ and

$$
\begin{aligned}
\left\|u_{j}-v_{j}\right\|_{W^{1, p(x)}(\Omega)} & \leq \frac{1}{n}, \\
E_{\lambda}\left(v_{j}\right) & \leq E_{\lambda}\left(u_{j}\right), \\
E_{\lambda}^{\prime}\left(v_{j}\right) & \longrightarrow 0 \quad \text { in }\left(W^{1, p(x)}(\Omega)\right)^{*}, \\
v_{j} & \longrightarrow u_{\lambda} \quad \text { weakly in } W^{1, p(x)}(\Omega) .
\end{aligned}
$$

Moreover, using the Brézis-Lieb lemma in [14] combined with the $p(x)$ version of Theorem 2.1 in Boccardo and Murat [15], it follows that $\nabla v_{j}(x) \rightarrow \nabla u_{\lambda}(x)$ for almost every $x \in \Omega$,

$$
\begin{aligned}
\int_{\Omega} \frac{\left|\nabla v_{j}\right|^{p(x)}}{p(x)} d x= & \int_{\Omega} \frac{\left|\nabla\left(v_{j}-u_{\lambda}\right)\right|^{p(x)}}{p(x)} \\
& +\int_{\Omega} \frac{\left|\nabla u_{\lambda}\right|^{p(x)}}{p(x)}+o_{j}(1),
\end{aligned}
$$




$$
\begin{aligned}
\int_{\Omega} f\left(x, v_{j}\right) d x= & \int_{\Omega} f\left(x, u_{\lambda}\right) d x \\
& +\int_{\Omega} f\left(x, v_{j}-u_{\lambda}\right) d x+o_{j}(1), \\
\int_{\Omega} \frac{\left|v_{j}\right|^{1-\delta(x)}}{1-\delta(x)} d x= & \int_{\Omega} \frac{\left|u_{\lambda}\right|^{1-\delta(x)}}{1-\delta(x)} d x \\
& +\int_{\Omega} \frac{\left|v_{j}-u_{\lambda}\right|^{1-\delta(x)}}{1-\delta(x)} d x+o_{j}(1) .
\end{aligned}
$$

Hence, from (28) and the compact boundary trace embedding in Theorem 5, we get $E_{\lambda}\left(v_{j}\right) \geq E_{\lambda}\left(u_{\lambda}\right)+o_{j}(1)$. Thus, it follows that $E_{\lambda}\left(u_{\lambda}\right)=c_{0}<0$. Hence $u_{\lambda} \neq \equiv$ and it is a local minimizer of $E_{\lambda}$ in $W^{1, p(x)}(\Omega)$.

We prove now the existence of positive solution to $\left(P_{\lambda}\right)$ for $0<\lambda<\Lambda$. Precisely, we have the following result.

Lemma 10. Problem $\left(P_{\lambda}\right)$ possesses a solution $u_{\lambda}$ for $0<\lambda<$ $\Lambda$.

Proof. Fix $0<\lambda<\lambda_{2}<\Lambda$. $\lambda_{2}$ such that there exist solutions to $\left(P_{\lambda}\right)$ for $\lambda=\lambda_{2}$, say $u_{\lambda_{2}}$. Note that $u_{\lambda_{2}}$ is a supersolution for $\left(P_{\lambda}\right)$. It is clear that 0 is not a local minimizer of $E_{\lambda}$ on $\left[0, u_{\lambda_{2}}\right] \cap$ $W^{1, p(x)}(\Omega)$ since $E_{\lambda}(0)=0$ and $E_{\lambda}(t v)<0$ for $t \rightarrow 0^{+}$. Now, we show the existence of a local minimizer of the functional energy. For this, we use the cut-off argument. Define

$$
\begin{aligned}
& \tilde{f}(x, s)= \begin{cases}f(x, 0), & \text { if } s<0, \\
f(x, s), & \text { if } 0 \leq s \leq u_{\lambda_{2}}, \\
f\left(x, u_{\lambda_{2}}\right), & \text { if } s>u_{\lambda_{2}}(x),\end{cases} \\
& \tilde{g}(x, s)= \begin{cases}0, & \text { if } s<0, \\
s^{-\delta(x)}, & \text { if } 0 \leq s \leq u_{\lambda_{2}}, \\
u_{\lambda_{2}}^{-\delta(x)}, & \text { if } s>u_{\lambda_{2}}(x),\end{cases} \\
& \tilde{h}(x, s)= \begin{cases}0, & \text { if } s<0, \\
s^{q(x)}, & \text { if } 0 \leq s \leq u_{\lambda_{2}}, \\
u_{\lambda_{2}}^{q(x)}, & \text { if } s>u_{\lambda_{2}}(x) .\end{cases}
\end{aligned}
$$

Also define the functional $\widetilde{E}_{\lambda}: W^{1, p(x)}(\Omega) \rightarrow \mathbb{R}$ by

$$
\begin{aligned}
\widetilde{E}_{\lambda}(u)= & \int_{\Omega} \frac{|\nabla u|^{p(x)}}{p(x)} d x+\int_{\Omega} \frac{|u|^{p(x)}}{p(x)} d x \\
& -\int_{\Omega} \widetilde{G}(x, u) d x-\int_{\Omega} \widetilde{F}(x, u) d x \\
& -\lambda \int_{\partial \Omega} \widetilde{H}(x, u) d x,
\end{aligned}
$$

where $\widetilde{F}(x, t)=\int_{0}^{t} \tilde{f}(x, s) d s, \widetilde{G}(x, t)=\int_{0}^{t} \widetilde{g}(x, s) d s$, and $\widetilde{H}(x, t)=\int_{0}^{t} \widetilde{h}(x, s) d s$. From the dominated convergence and the compact boundary trace embedding in Theorem 5, it is easy to see that $\widetilde{E}_{\lambda}$ is bounded below and is weakly lower semicontinuous in $W^{1, p(x)}(\Omega)$. Then, there exists $\widetilde{u}_{\lambda} \epsilon$ $W^{1, p(x)}(\Omega)$ such that $\widetilde{E}_{\lambda}$ achieves its global minimum in $W^{1, p(x)}(\Omega)$. Moreover, since $\widetilde{E}_{\lambda}$ is $C^{1}\left(W^{1, p(x)}(\Omega), \mathbb{R}\right)$ from Lemma A.4, $\tilde{u}_{\lambda}$ solves the equation

$$
\begin{array}{rr}
-\Delta_{p(x)} \widetilde{u}_{\lambda}+\left|\widetilde{u}_{\lambda}\right|^{p(x)-2} \widetilde{u}_{\lambda}=\widetilde{g}\left(x, \tilde{u}_{\lambda}\right)+\tilde{f}\left(x, \tilde{u}_{\lambda}\right), & \text { in } \Omega, \\
\left|\nabla \widetilde{u}_{\lambda}\right|^{p(x)-2} \frac{\partial \tilde{u}_{\lambda}}{\partial \nu}=\lambda \widetilde{h}\left(x, \tilde{u}_{\lambda}\right), & \text { on } \partial \Omega .
\end{array}
$$

Now, using the strong maximum principle (see Theorem 8) $\widetilde{u}_{\lambda} \not \equiv 0$ and $\widetilde{u}_{\lambda}>0$ since 0 is not a local minimizer of $E_{\lambda}$ on $\left[0, u_{\lambda_{2}}\right] \cap W^{1, p(x)}(\Omega)$. By the definition of $\tilde{f}, \tilde{g}$, and $\tilde{h}$ we have

$$
\begin{array}{ll}
\widetilde{g}(x, 0) \leq \widetilde{g}\left(x, \widetilde{u}_{\lambda}\right) \leq \widetilde{g}\left(x, u_{\lambda_{2}}\right), & \forall x \in \Omega, \\
\widetilde{f}(x, 0) \leq \widetilde{f}\left(x, \widetilde{u}_{\lambda}\right) \leq \widetilde{f}\left(x, u_{\lambda_{2}}\right), & \forall x \in \Omega, \\
\widetilde{h}(x, 0) \leq \widetilde{h}\left(x, \tilde{u}_{\lambda}\right) \leq \widetilde{h}\left(x, u_{\lambda_{2}}\right), \quad \forall x \in \Omega .
\end{array}
$$

Again, by the strong comparison principle (see Lemma 7), we conclude that $\widetilde{u}_{\lambda} \leq u_{\lambda_{2}}$ in $\Omega$ and $(\partial / \partial \nu)\left(u_{\lambda_{2}}-\widetilde{u}_{\lambda}\right) \leq 0$ on $\partial \Omega$. Hence $\tilde{u}_{\lambda}$ is a solution to $\left(P_{\lambda}\right)$. This completes the proof of Lemma 10.

Now, we show the following result.

Lemma 11. There exists at least one positive weak solution for $\lambda=\Lambda$ to problem $\left(P_{\lambda}\right)$.

Proof. Let $\left\{\lambda_{k}\right\}_{k \in \mathbb{N}} \in(0, \Lambda), \lambda_{k} \uparrow \Lambda$ as $k \rightarrow \infty$, and $u_{\lambda_{k}}$ be a solution of $\left(P_{\lambda_{k}}\right)$ such that $u_{\lambda_{k-1}} \leq u_{\lambda_{k}}$ for all $x \in \Omega$. Now, taking $u_{\lambda_{k}}$ as test function in $\left(P_{\lambda_{k}}\right)$, we get

$$
\begin{aligned}
& \int_{\Omega}\left|\nabla u_{\lambda_{k}}\right|^{p(x)} d x+\int_{\Omega}\left|u_{\lambda_{k}}\right|^{p(x)} d x \\
& =\int_{\Omega} u_{\lambda_{k}}^{1-\delta(x)} d x+\int_{\Omega} f\left(x, u_{\lambda_{k}}\right) u_{\lambda_{k}} d x \\
& \quad+\lambda_{k} \int_{\partial \Omega} u_{\lambda_{k}}^{q(x)+1} d x
\end{aligned}
$$

Moreover, as $E_{\lambda_{k}}\left(u_{\lambda_{k}}\right) \leq E_{\lambda_{k}}(0)=0$, we have

$$
\begin{gathered}
\int_{\Omega} \frac{\left|\nabla u_{\lambda_{k}}\right|^{p(x)}}{p(x)} d x+\int_{\Omega} \frac{\left|u_{\lambda_{k}}\right|^{p(x)}}{p(x)} d x-\int_{\Omega} \frac{u_{\lambda_{k}}^{1-\delta(x)}}{1-\delta(x)} d x \\
-\int_{\Omega} F\left(x, u_{\lambda_{k}}\right) d x-\lambda_{k} \int_{\partial \Omega} \frac{u_{\lambda_{k}}^{q(x)+1}}{q(x)+1} d x \leq 0 .
\end{gathered}
$$


It follows that

$$
\begin{aligned}
& \frac{1}{p^{+}}\left(\int_{\Omega}\left|\nabla u_{\lambda_{k}}\right|^{p(x)} d x+\int_{\Omega}\left|u_{\lambda_{k}}\right|^{p(x)} d x\right) \\
& \leq \int_{\Omega} u_{\lambda_{k}}^{1-\delta(x)} d x+\int_{\Omega} F\left(x, u_{\lambda_{k}}\right) d x \\
& \quad+\lambda_{k} \int_{\partial \Omega} u_{\lambda_{k}}^{q(x)+1} d x .
\end{aligned}
$$

Now, from (7) there exists $C>0$ such that, for $\theta>p_{+}$and for all $t>0$,

$$
\theta F(x, t) \leq t f(x, t)+C .
$$

Moreover, using Theorem 4.2 in [16], we get the existence of the constants $C_{1}>0, C_{2}>0$ such that

$$
C_{1}[d(x)]^{\theta_{1}} \leq \underline{u}_{\lambda}(x) \leq C_{2}[d(x)]^{\theta_{2}},
$$

where $\theta_{1}=\max _{d(x) \leq \sigma}(p(x) /(p(x)-1+\delta(x))), \theta_{2}=$ $\min _{d(x) \leq \sigma}(p(x) /(p(x)-1+\delta(x)))$, and $d(x)=\operatorname{dist}(x, \partial \Omega)$. On the other hand, we recall the following inequality due to Lieberman [17]. There exists a constant $K(\Omega)>0$ such that

$$
\int_{\partial \Omega}|u| \leq \int_{\Omega}|\nabla u|+K(\Omega) \int_{\Omega}|u|, \quad \forall u \in W^{1,1}(\Omega) .
$$

Inserting (36), (37), and (38) in (33), we get $\sup _{k \in \mathbb{N}}$ $\left\|u_{\lambda_{k}}\right\|_{W^{1, p(x)}(\Omega)}<\infty$. It follows that $\left\{u_{\lambda_{k}}\right\}$ is bounded in $W^{1, p(x)}(\Omega)$ since $u_{\lambda_{k}}(x) \geq u_{\lambda_{1}}(x)$ for all $x \in \Omega$. Without loss of generality, $u_{\lambda_{k}} \rightarrow u_{\Lambda}$ in $W^{1, p(x)}(\Omega)$ and then by the Sobolev imbedding $u_{\lambda_{k}} \rightarrow u_{\Lambda}$ in $L^{q(x)}(\Omega)$ and $u_{\lambda_{k}(x)} \rightarrow u_{\Lambda}(x)$ for a.e. $x \in \Omega$. By the $L^{\infty}(\Omega)$-regularity results of [13], the boundedness of $\left\{\left\|u_{\lambda_{k}}\right\|\right\}$ implies the boundedness of $\left\{\left|u_{\lambda_{k}}\right|_{L^{\infty}(\Omega)}\right\}$. By the $C^{1, \alpha}(\Omega)$-regularity Theorem 16, the boundedness of $\left\{\left|u_{\lambda_{k}}\right|_{L^{\infty}}(\Omega)\right\}$ implies the boundedness of $\left\{\left\|u_{\lambda_{k}}\right\|_{C^{1, \alpha}(\bar{\Omega})}\right\}$, where $\alpha \in(0,1)$ is a constant. Thus, we have $u_{\lambda_{k}} \rightarrow u_{\Lambda}$ in $C^{1}(\bar{\Omega})$. For every $v \in W^{1, p(x)}(\Omega)$, since $u_{\lambda_{k}}$ is a solution of problem $\left(P_{\lambda_{k}}\right)$, we have that, for each $k$,

$$
\begin{gathered}
\int_{\Omega}\left|\nabla u_{\lambda_{k}}\right|^{p(x)-2} \nabla u_{\lambda_{k}} \nabla v d x+\int_{\Omega}\left|u_{\lambda_{k}}\right|^{p(x)-2} u_{\lambda_{k}} v d x \\
=\int_{\Omega}\left|u_{\lambda_{k}}\right|^{-\delta(x)} v d x+\int_{\Omega} f\left(x, u_{\lambda_{k}}\right) v d x \\
+\lambda_{k} \int_{\partial \Omega}\left|u_{\lambda_{k}}\right|^{q(x)} v d x .
\end{gathered}
$$

Passing to the limit in (39) as $k \rightarrow \infty$ yields

$$
\begin{aligned}
& \int_{\Omega}\left|\nabla u_{\Lambda}\right|^{p(x)-2} \nabla u_{\Lambda} \nabla v d x+\int_{\Omega}\left|u_{\Lambda}\right|^{p(x)-2} u_{\Lambda} v d x \\
& =\int_{\Omega} u_{\Lambda}^{-\delta(x)} v d x+\int_{\Omega} f\left(x, u_{\Lambda}\right) v d x \\
& \quad+\Lambda \int_{\partial \Omega} u_{\Lambda}^{q(x)} v d x,
\end{aligned}
$$

which shows that $u_{\Lambda}$ is a solution of $\left(P_{\Lambda}\right)$. Obviously $u_{\Lambda} \geq 0$ and $u_{\Lambda} \not \equiv 0$. Hence $u_{\Lambda}$ is a positive solution of $\left(P_{\Lambda}\right)$ in $W^{1, p(x)}(\Omega)$. This completes the proof of Lemma 11 .

Then we prove the following nonexistence result.
Lemma 12. Consider the following:

$\Lambda<\infty$.

Proof. Let $u_{\lambda}$ be a solution of $\left(P_{\lambda}\right)$. Taking $\varphi \equiv 1$ as a test function in the weak formulation of $\left(P_{\lambda}\right)$, we get

$$
\begin{aligned}
\int_{\Omega} u_{\lambda}^{p(x)-1} d x= & \int_{\Omega} u_{\lambda}^{-\delta(x)} d x+\int_{\Omega} f\left(x, u_{\lambda}\right) d x \\
& +\lambda \int_{\partial \Omega} u_{\lambda}^{q(x)} d x .
\end{aligned}
$$

On the other hand, we have

$$
\int_{\Omega} u_{\lambda}^{p(x)-1} \leq \int_{\Omega} u_{\lambda}^{p^{+}-1}+C_{1} .
$$

Using assumption (H2) we have $C_{2} \int_{\Omega} u_{\lambda}^{-\delta^{+}} \leq \int_{\Omega} u^{-\delta(x)}$, and using (6) we have $C_{3} \int_{\Omega} u_{\lambda}^{\gamma} \leq \int_{\Omega} f(x, u) d x$. Therefore, from (41) and (42) we get

$$
\int_{\Omega} u_{\lambda}^{\gamma} \leq C_{3} \int_{\Omega} u_{\lambda}^{p^{+}}-C_{4} \int_{\Omega} u_{\lambda}^{-\delta^{+}}+C_{5} .
$$

Now, since $p^{+}-1<\gamma$ and $-\delta^{+}<\gamma$, by the embedding of $L^{\gamma}(\Omega)$ into $L^{p^{+}-1}(\Omega)$ and by the embedding of $L^{\gamma}(\Omega)$ into $L^{-\delta^{+}}(\Omega)$ we obtain

$$
\begin{gathered}
\int_{\Omega} u_{\lambda}^{p^{+}-1} \leq C_{6}\left(\int_{\Omega} u_{\lambda}^{\gamma}\right)^{\left(p^{+}-1\right) / \gamma}, \\
\int_{\Omega} u_{\lambda}^{-\delta^{+}} \leq C_{7}\left(\int_{\Omega} u_{\lambda}^{\gamma}\right)^{-\delta^{+} / \gamma} .
\end{gathered}
$$

Substituting (44) in (43) we get

$$
\left\|u_{\lambda}\right\|_{L^{\gamma}(\Omega)}^{\gamma} \leq C_{8}+C_{9}\left\|u_{\lambda}\right\|_{L^{\gamma}(\Omega)}^{p^{+}-1}+C_{10}\left\|u_{\lambda}\right\|_{L^{\gamma}(\Omega)}^{\delta^{+}} .
$$

Hence, $\left\|u_{\lambda}\right\|_{L^{\gamma}(\Omega)}$ is bounded by a constant independent of $\lambda$. Now, taking $\varphi=u_{\lambda}^{-q^{-}}$as a test function in the weak formulation of $\left(P_{\lambda}\right)$ and using $(\mathbf{H} 2)$ we get

$$
\begin{aligned}
\int_{\Omega}\left|u_{\lambda}\right|^{p(x)-1-q^{-}} d x \geq & \int_{\Omega} u_{\lambda}^{-\delta(x)-q^{-}} d x \\
& +\int_{\Omega} f\left(x, u_{\lambda}\right) u_{\lambda}^{-q^{-}} d x \\
& +\lambda \int_{\partial \Omega} u_{\lambda}^{q(x)-q^{-}} d x \\
\geq & \lambda \int_{\partial \Omega} u_{\lambda}^{q(x)-q^{-}} d x \\
\geq & \lambda \int_{\Gamma} u_{\lambda}^{q^{-}-q^{-}} d x=\lambda|\Gamma|_{N-1} .
\end{aligned}
$$

Now since

$$
\int_{\Omega}\left|u_{\lambda}\right|^{p(x)-1-q^{-}} d x \leq C+\left\|u_{\lambda}\right\|_{L^{\gamma}(\Omega)}^{\gamma}
$$

it follows that $\Lambda$ is finite. The proof of Lemma 12 is now completed.

Proof of Theorem 2. Theorem 2 follows from Lemmas 10, 11, and 12. 
Now, we prove that the solution of problem $\left(P_{\lambda}\right)$ obtained in Lemma 10 is a local minimum for the functional energy associated to problem $\left(P_{\lambda}\right)$. Precisely, we have the following result.

Lemma 13. Let $u_{\lambda}$ be the weak solution of problem $\left(P_{\lambda}\right)$ obtained in Lemma 10. Then, $u_{\lambda}$ is a local minimum for $E_{\lambda} \backslash_{C^{1}(\bar{\Omega})}$.

Proof. Fix $0<\lambda_{1}<\lambda<\lambda_{2}<\Lambda$ and let $u_{\lambda_{1}}, u_{\lambda_{2}}$ be solutions to $\left(P_{\lambda}\right)$ for $\lambda=\lambda_{1}$ and $\lambda=\lambda_{2}$, respectively, such that $u_{\lambda_{1}} \leq$ $u_{\lambda} \leq u_{\lambda_{2}}$. By Lemma 7, $u_{\lambda_{1}}<u_{\lambda}<u_{\lambda_{2}}$ on $\bar{\Omega}$. Define the following cut-off functions:

$$
\begin{aligned}
& \hat{f}(x, t)= \begin{cases}f\left(x, u_{\lambda_{1}}(x)\right), & t<u_{\lambda_{1}}(x), \\
f(x, t), & u_{\lambda_{1}}(x) \leq t \leq u_{\lambda_{2}}(x), \\
f\left(x, u_{\lambda_{2}}(x)\right), & t>u_{\lambda_{2}}(x),\end{cases} \\
& \hat{g}(x, t)= \begin{cases}u_{\lambda_{1}(x)}^{-\delta(x)}, & t<u_{\lambda_{1}}(x), \\
t^{-\delta(x)}, & u_{\lambda_{1}}(x) \leq t \leq u_{\lambda_{2}}(x), \\
u_{\lambda_{2}}^{-\delta(x)}, & t>u_{\lambda_{2}}(x),\end{cases}
\end{aligned}
$$$$
(x \in \Omega, t \in \mathbb{R}),
$$$$
\widehat{h}(x, t)= \begin{cases}\psi(x) u_{\lambda_{1}}^{q(x)}, & t<u_{\lambda_{1}}(x), \\ \psi(x) t^{q(x)}, & u_{\lambda_{1}}(x) \leq t \leq u_{\lambda_{2}}(x), \\ \psi(x) u_{\lambda_{2}}^{q(x)}, & t>u_{\lambda_{2}}(x),\end{cases}
$$

$(x \in \partial \Omega, t \in \mathbb{R})$.

Then the corresponding functional $\widehat{E}_{\lambda}: W^{1, p(x)}(\Omega) \rightarrow \mathbb{R}$ given by

$$
\begin{aligned}
\widehat{E}_{\lambda}(u)= & \int_{\Omega} \frac{|\nabla u|^{p(x)}}{p(x)}+\int_{\Omega} \frac{|u|^{p(x)}}{p(x)}-\int_{\Omega} \widehat{G}(x, u) \\
& -\int_{\Omega} \widehat{F}(x, u)-\lambda \int_{\partial \Omega} \widehat{H}(x, u),
\end{aligned}
$$

where $\widehat{F}(x, u)=\int_{0}^{u} \widehat{f}(x, t) d t(x \in \Omega), \widehat{G}(x, u)=\int_{0}^{u} \widehat{g}(x$, $t) d t(x \in \Omega)$, and $\widehat{H}(x, u)=\int_{0}^{u} \widehat{h}(x, t) d t(x \in \partial \Omega)$. Firstly, from Lemma A.4 $\widehat{E}_{\lambda}$ is $C^{1}\left(W^{1, p(x)}(\Omega), \mathbb{R}\right)$. Then, it is simple to see that $\widehat{E}_{\lambda}$ is coercive and bounded below. Let $u_{\lambda}$ denote the global minimum of $\widehat{E}_{\lambda}$ on $W^{1, p(x)}(\Omega)$ which satisfies the equation

$$
\begin{array}{r}
-\operatorname{div}\left(|\nabla u|^{p(x)-2} \nabla u\right)+|u|^{p(x)-2} u \\
=\hat{g}(x, u)+\widehat{f}(x, u), \quad u>0 \text { in } \Omega, \\
|\nabla u|^{p(x)-2} \frac{\partial u}{\partial \nu}=\lambda \widehat{h}(x, u), \quad \text { on } \partial \Omega .
\end{array}
$$

Therefore, from the regularity results Theorem 16, we conclude that $u_{\lambda}$ is in $C^{1, \alpha}(\bar{\Omega})$ for some $0<\alpha<1$. Now, using (H2) and since $f(x, t)$ is nondecreasing, by the definition of $\widehat{f}, \widehat{g}$, and $\widehat{h}$ we have

$$
\begin{array}{ll}
\widehat{g}\left(x, u_{\lambda_{1}}\right) \leq \hat{g}\left(x, u_{\lambda}\right) \leq \hat{g}\left(x, u_{\lambda_{2}}\right), & \forall x \in \Omega, \\
\widehat{f}\left(x, u_{\lambda_{1}}\right) \leq \widehat{f}\left(x, u_{\lambda}\right) \leq \widehat{f}\left(x, u_{\lambda_{2}}\right), & \forall x \in \Omega, \\
\widehat{h}\left(x, u_{\lambda_{1}}\right) \leq \widehat{h}\left(x, u_{\lambda}\right) \leq \widehat{h}\left(x, u_{\lambda_{2}}\right), & \forall x \in \partial \Omega .
\end{array}
$$

Again, by the strong comparison principle (see Lemma 7), we conclude that $u_{\lambda_{1}} \leq u_{\lambda} \leq u_{\lambda_{2}}$ in $\Omega$. Let $\beta=\inf _{x \in \bar{\Omega}}\left\{\mid u_{\lambda_{1}}-\right.$ $\left.u_{\lambda_{2}}|+| \nabla u_{\lambda_{1}}-\nabla u_{\lambda_{2}} \mid\right\}$. We claim that $\beta>0$. If not, then there exists $x \in \partial \Omega$ such that $u_{\lambda_{1}}=u_{\lambda_{2}}$ and $\nabla u_{\lambda_{1}}=\nabla u_{\lambda_{2}}$. But this contradicts the boundary data as $\lambda_{1} \neq \lambda_{2}$. Therefore, if $v \in$ $\mathscr{C}=\left\{u \in C^{1}(\bar{\Omega}) \backslash\left\|u-u_{\lambda}\right\|_{C^{1}(\bar{\Omega})}<\beta / 2\right\}$ then $u_{\lambda_{1}}<u_{\lambda}<u_{\lambda_{2}}$ in $\bar{\Omega}$ since $E_{\lambda} \equiv \widehat{E}_{\lambda}$ on the set $\mathscr{C}$. Hence $u_{\lambda}$ is a local minimum for $E_{\lambda} l_{C^{1}(\bar{\Omega})}$. This completes the proof of Lemma 13 .

\section{4. $C^{1}$ versus $W^{1, p(x)}$ Local Minimizers of the Energy}

The following lemma is crucial in showing multiplicity of solutions. It has been shown in the case $p=2$ in [18] for the case of critical growth functionals $E_{\lambda}: H_{0}^{1}(\Omega) \rightarrow \mathbb{R}$, $\Omega \subset \mathbb{R}^{N}, N \geq 3$ and later for critical growth functionals $E_{\lambda}: W_{0}^{1, p}(\Omega) \rightarrow \mathbb{R}, 1<p<N, \Omega \subset \mathbb{R}^{N}, N \geq 3$ in [19]. A key feature of these latter works is the uniform $C^{1, \alpha}$ estimate they obtain for equations like $\left(P_{\epsilon}\right)$ but involving two $p$-Laplace operators. Using constraints based on $L^{p}$-norms rather than Sobolev norms as in [19], the equations for which uniform estimates are required can be simplified to a standard type involving only one $p$-Laplace operator. This approach was followed in [20] in the subcritical case, in [21] in the critical case, in [22-24], and also in this work to deal with the boundary value problem involving the nonlinear $p(x)$ Laplacian case. More precisely, we have the following result.

Lemma 14. Suppose that conditions (H0)-(H5) are satisfied. Let $u_{0} \in C^{1}(\bar{\Omega})$ satisfying

$$
u_{0} \geq \eta d(x, \partial \Omega) \text { for some } \eta>0
$$

be a local minimizer of $E_{\lambda}$ in $C^{1}(\bar{\Omega}) \cap C_{0}(\bar{\Omega})$ topology. Then, $u_{0}$ is a local minimum of $E_{\lambda}$ in $W^{1, p(x)}(\Omega)$ also.

For proving Lemma 14, we will need the following uniform $L^{\infty}$-estimates for a family of solutions to $\left(P_{\epsilon}\right)$.

Proposition 15. Let $\left\{u_{\epsilon}\right\}_{\epsilon \in(0,1)}$ be a family of solutions to $\left(P_{\epsilon}\right)$, where $u_{0}$ satisfies (52) and solves $\left(P_{\lambda}\right)$. Let $\gamma>1$ be such that

$$
\sup _{\epsilon \in(0,1)}\left(\left\|f\left(x, u_{\epsilon}\right)\right\|_{L^{\gamma}}+\left\|u_{\epsilon}\right\|_{W^{1, p(x)}(\Omega)}\right)<\infty .
$$

Then,

$$
\left\|u_{\epsilon}\right\|_{L^{\infty}(\Omega)}<C
$$


The proof of Proposition 15 is a consequence of the results proved in Appendix A in [13]. Hence, the regularity results of Saoudi and Ghanmi [13] give the following regularity result for weak solutions to problem $\left(P_{\lambda}\right)$.

Theorem 16. Let $u \in W^{1, p(x)}(\Omega) \cap L^{\infty}(\Omega)$ be a solution to problem $\left(P_{\lambda}\right)$. Then, there exists $0<K=K\left(\|u\|_{L^{\infty}(\Omega)}\right.$, $\left.p^{+}, p^{-}, \delta^{+}, \delta^{-}, \Omega, N\right)$ such that any weak solution to problem $\left(P_{\lambda}\right)$ belongs to $C^{1, \alpha}(\bar{\Omega})$ for some $\alpha \in(0,1)$.

Proof of Lemma 14. Assume that the conclusion of Lemma 14 is not true. We define the following constraint for each $\epsilon>0$ :

$$
\begin{aligned}
\mathcal{S}_{\epsilon} & \stackrel{\text { def }}{=}\left\{u \in W^{1, p(x)}(\Omega): \rho(u) \stackrel{\text { def }}{=} \int_{\Omega} \frac{u^{\alpha(x)}}{\alpha(x)}\right. \\
& \left.+\frac{1}{p^{-}} \int_{\partial \Omega} u^{p^{-}} \leq \epsilon\right\} .
\end{aligned}
$$

We consider the following constraint minimization problem:

$$
I_{\epsilon}=\inf _{u \in \mathcal{S}_{\epsilon}} E_{\lambda}(u)
$$

Firstly, clearly $I_{\epsilon}>-\infty$. Moreover, we note that $\mathcal{S}_{\epsilon}$ is a convex set. Using the trace embeddings we see that $\mathcal{S}_{\epsilon}$ is also a closed set in $W^{1, p(x)}(\Omega)$ which implies that $\mathcal{S}_{\epsilon}$ is weakly closed in $W^{1, p(x)}(\Omega)$; with the fact that $E_{\lambda}$ is weakly low semicontinuous in $W^{1, p(x)}(\Omega)$, it follows that for $\epsilon \in(0,1) I_{\epsilon}$ is achieved on some $u_{\epsilon} \in \mathcal{S}_{\epsilon}$; that is

$$
\begin{aligned}
& E_{\lambda}\left(u_{\epsilon}\right)=I_{\epsilon}, \\
& E_{\lambda}\left(u_{\varepsilon}\right)<E_{\lambda}\left(u_{0}\right)
\end{aligned}
$$

$\forall \epsilon \in(0,1)$.

Moreover, since $E_{\lambda}\left(u_{\epsilon}^{+}\right) \leq E_{\lambda}\left(u_{\epsilon}\right)$ and $u_{\epsilon}^{+} \in \mathcal{S}_{\epsilon}$, we may assume that $u_{\epsilon} \geq 0$.

We now consider the following two cases.

(1) Let $\rho\left(u_{\epsilon}\right)<\epsilon$. Then $u_{\epsilon}$ is also a local minimizer of $E_{\lambda}$ in $W^{1, p(x)}(\Omega)$. We now show that $E_{\lambda}$ admits Gâteaux derivatives on $u_{\epsilon}$ to derive that $u_{\epsilon}$ satisfies the Euler-Lagrange equation associated with $E_{\lambda}$. For this, according to Lemma A.2, in the Appendix, we need to prove that $\exists \widetilde{\eta}>0$ such that

$$
u_{\epsilon} \geq \tilde{\eta} d(x) \operatorname{dist}(x, \partial \Omega),
$$

where $d(x) \stackrel{\text { def }}{=} \operatorname{dist}(x, \partial \Omega)$. To prove (58), we argue by contradiction: $\forall \eta>0$ let

$$
\Omega_{\eta}=\operatorname{Supp}\left\{\left(\eta d(x)-u_{\epsilon}\right)^{+}\right\}
$$

and suppose that $\Omega_{\eta}$ has a nonzero measure.

Let $u_{\eta}=\left(\eta d(x)-u_{\epsilon}\right)^{+}$and for $0<t \leq 1$ set $\xi(t)=$ $E_{\lambda}\left(u_{\epsilon}+t u_{\eta}\right)$. Then, there exists $c(t)$ satisfying $c(t)>\eta t$ such that $\inf \left(\left(u_{\epsilon}+t u_{\eta}\right) / d(x)\right) \geq c(t)$ for $t>0$. Then, from Lemma A.4 $\xi$ is differentiable for $0<t \leq 1$ and $\xi^{\prime}(t)=$ $\left\langle E_{\lambda}^{\prime}\left(u_{\epsilon}+t u_{\eta}\right), u_{\eta}\right\rangle$. Thus,

$$
\xi^{\prime}(t)=\int_{\Omega}\left|\nabla\left(u_{\epsilon}+t u_{\eta}\right)\right|^{p(x)-2} \nabla\left(u_{\epsilon}+t u_{\eta}\right) \nabla u_{\eta}
$$

$$
\begin{aligned}
& +\int_{\Omega}\left|u_{\epsilon}+t u_{\eta}\right|^{p(x)-2} u_{\eta} \\
& -\int_{\Omega}\left(u_{\epsilon}+t u_{\eta}\right)^{-\delta(x)} u_{\eta} \\
& -\int_{\Omega} f\left(x, u_{\epsilon}+t u_{\eta}\right) u_{\eta} \\
& -\lambda \int_{\Omega}\left|u_{\epsilon}+t u_{\eta}\right|^{q(x)-1}\left(u_{\epsilon}+t u_{\eta}\right) u_{\eta} .
\end{aligned}
$$

From (H1)-(H3), we see that

$$
\begin{aligned}
\xi^{\prime}(1)= & \int_{\Omega}|\nabla \eta d(x)|^{p(x)-2} \nabla(\eta d(x)) \nabla u_{\eta} \\
& +\int_{\Omega}|\eta d(x)|^{p(x)-2} u_{\eta}-\int_{\Omega}(\eta d(x))^{-\delta(x)} u_{\eta} \\
& -\int_{\Omega} f(x, \eta d(x)) u_{\eta} \\
& -\lambda \int_{\Omega}|\eta d(x)|^{q(x)-1}(\eta d(x)) u_{\eta}<0,
\end{aligned}
$$

for $\eta>0$ small enough.

Now, since $s^{-\delta(x)}+f(x, s)+\lambda s^{q(x)}$ is nonincreasing for $0<$ $s$ small enough uniformly to $x \in \Omega$ (by (H1)-(H3)) and from the monotonicity of the operator $-\Delta_{p(x)} u+|u|^{p(x)-1} u$, we have that for $0<\eta$ small enough $0 \leq \xi^{\prime}(1)-\xi^{\prime}(t)$. Therefore, from Taylor's expansion and since $\rho\left(u_{\epsilon}\right) \leq \epsilon$, there exists $0<\gamma<1$ such that

$$
\begin{aligned}
0 & \leq E_{\lambda}\left(u_{\epsilon}+u_{\eta}\right)-E_{\lambda}\left(u_{\epsilon}\right)=\left\langle E_{\lambda}^{\prime}\left(u_{\epsilon}+\gamma u_{\eta}\right), u_{\eta}\right\rangle \\
& =\xi^{\prime}(\gamma) .
\end{aligned}
$$

Letting $t=\gamma$ we have $\xi^{\prime}(\gamma) \leq \xi^{\prime}(1)<0$. We obtain a contradiction with (62) and then $u_{\epsilon} \geq \eta d(x)$ for some $\eta>0$ (which depends a priori on $\epsilon$ ). Since $u_{\epsilon}$ is a local minimizer of $E_{\lambda}$ and $E_{\lambda}$ is Gâteaux differentiable in $u_{\epsilon}$, we get that $E_{\lambda}^{\prime}\left(u_{\epsilon}\right)$ is defined and $E_{\lambda}^{\prime}\left(u_{\epsilon}\right)=0$. Recalling that $\underline{u}$ is the solution to the pure singular problem given by Theorem 4.2 in [16] and from the weak comparison principle, there exist $C_{1}>0, C_{2}>0$ such that

$$
C_{1}[d(x)]^{\theta_{1}} \leq \underline{u} \leq u_{\epsilon}
$$

where $\theta_{1}=\max _{d(x) \leq \sigma}(p(x) /(p(x)-1+\delta(x)))$, for some $\eta>$ 0 (independent of $\epsilon$ ). Since $u_{\epsilon} \in \mathcal{S}_{\epsilon}$ and from the fact that $u_{\epsilon}$ satisfies $\left(P_{\lambda}\right)$, we get that $\left\{u_{\epsilon}\right\}_{\epsilon \geq 0}$ is uniformly bounded in $W^{1, p(x)}(\Omega)$. Now, using Proposition 15 and Theorem 16, we get

$$
\left|u_{\epsilon}\right|_{C^{1, \alpha}(\bar{\Omega})} \leq C \quad \text { for some } \alpha \in(0,1)
$$

and as $\epsilon \rightarrow 0^{+}$

$$
u_{\epsilon} \longrightarrow u_{0} \quad \text { in } C^{1}(\bar{\Omega})
$$

which contradicts the fact that $u_{0}$ is a local minimizer in $C^{1}(\bar{\Omega}) \cap C_{0}(\bar{\Omega})$. 
Now, we deal with the second case.

(2) $\rho\left(u_{\epsilon}\right)=\epsilon$ : we again show that $u_{\epsilon} \geq \eta d(x)$ in $\Omega$ for some $\eta>0$. Taking $u_{\eta}=\left(\eta d(x)-u_{\epsilon}\right)^{+}, \xi(t)=E_{\lambda}\left(u_{\epsilon}+t u_{\eta}\right)$, we obtain as above that $\xi^{\prime}(t) \leq \xi^{\prime}(1)<0$ for $0<t<1$ and $0<\eta$ small enough.

Then $\xi(t)=E_{\lambda}\left(u_{\epsilon}+t u_{\eta}\right)$ is decreasing. This implies that $E_{\lambda}\left(u_{\epsilon}\right)>E_{\lambda}\left(u_{\epsilon}+t u_{\eta}\right)$ for $t>0$ and using (52)

$$
\rho\left(u_{\epsilon}+t u_{\eta}\right)<\rho\left(u_{\epsilon}\right)=\epsilon .
$$

This yields a contradiction with the fact that $u_{\epsilon}$ is a global minimizer of $E_{\lambda}$ on $\mathcal{S}_{\epsilon}$. In this case, using Lemma A.4 and from the Lagrange multiplier rule we have

$$
E_{\lambda}^{\prime}\left(u_{\epsilon}\right)=\mu_{\epsilon} \rho^{\prime}\left(u_{\epsilon}\right), \text { for some } \mu_{\epsilon} \in \mathbb{R}
$$

We first show that $\mu_{\epsilon} \leq 0$. We argue by contradiction. Suppose that $\mu_{\epsilon}>0$; then there exists $\varphi \in W^{1, p(x)}(\Omega)$ such that

$$
\begin{aligned}
& \left\langle E_{\lambda}^{\prime}\left(u_{\epsilon}\right), \varphi\right\rangle<0, \\
& \left\langle\rho^{\prime}\left(u_{\epsilon}\right), \varphi\right\rangle<0
\end{aligned}
$$

and then for $t$ small we have

$$
\begin{aligned}
& E_{\lambda}\left(u_{\epsilon}+t \varphi\right)<E_{\lambda}\left(u_{\epsilon}\right), \\
& \rho\left(u_{\epsilon}+t \varphi\right)<\rho\left(u_{\epsilon}\right) \leq \epsilon .
\end{aligned}
$$

This contradicts the fact that $u_{\epsilon}$ is a global minimizer of $E_{\lambda}$ in $\mathcal{S}_{\epsilon}$.

We deal now with the two following cases.

Case $1\left(\inf _{\epsilon \in(0,1)} \mu_{\epsilon} \stackrel{\text { def }}{=} l>-\infty\right)$. In this case, we write (67) in its PDE form as

$$
\left(P_{\epsilon}\right) \begin{cases}-\Delta_{p(x)} u_{\varepsilon}+\left|u_{\varepsilon}\right|^{p(x)-1} u_{\epsilon}=\frac{1}{\left|u_{\varepsilon}\right|^{\delta(x)}+f\left(x, u_{\varepsilon}\right)+\mu_{\varepsilon}\left|u_{\varepsilon}\right|^{\alpha(x)-2} u_{\varepsilon},} & u_{\varepsilon}>0 \text { in } \Omega, \\ \left|\nabla u_{\varepsilon}\right|^{p(x)-2} \frac{\partial u_{\varepsilon}}{\partial \nu}=\lambda u_{\varepsilon}^{q(x)}+\mu_{\varepsilon}\left|u_{\varepsilon}\right|^{p^{-}-2} u_{\varepsilon}, & \text { on } \partial \Omega .\end{cases}
$$

In this case, from (57), we have that $\sup _{\epsilon \in(0,1)}\left\|u_{\epsilon}\right\|_{W^{1, p(x)}(\Omega)}$ $<\infty$. Hence, we can apply Proposition 15 to conclude that $\sup _{\epsilon \in(0,1)}\left\|u_{\epsilon}\right\|_{L^{\infty}(\Omega)}<K$ for some constant $K>0$ independent of $\epsilon$. Therefore, using Theorem 16 we conclude that $\left|u_{\epsilon}\right|_{C^{1, \alpha}(\bar{\Omega})} \leq C$ for some constant $C>0$ independent of $\epsilon$ and as $\epsilon \rightarrow 0^{+}$

$$
u_{\epsilon} \longrightarrow u_{0} \quad \text { in } C^{1}(\bar{\Omega})
$$

which contradicts the fact that $u_{0}$ is a local minimizer in $C^{1}(\bar{\Omega}) \cap C_{0}(\bar{\Omega})$.

Now, we deal with the second case.

Case $2\left(\inf _{\epsilon \in(0,1)} \mu_{\epsilon}=-\infty\right)$. From above, we can assume that $\mu_{\epsilon} \leq-1$ for $0<\epsilon$ small enough. Furthermore, we can find a number $M>0$ independent of $\epsilon>0$ and $x \in \bar{\Omega}$, such that $\left(1 / s^{\delta(x)}+f(x, s)+\mu_{\varepsilon}|s|^{\alpha(x)-2} s\right)$ and $\left(|s|^{q(x)}+\mu_{\varepsilon}|s|^{p^{-}-2} s\right) s$ are negative for all $|s| \geq M$. Then, from the weak comparison principle (see Lemma 7 and using $\left(u_{\varepsilon}-M\right)^{+}$as test function) we have that $\sup _{\epsilon \in(0,1)}\left\|u_{\epsilon}\right\|_{L^{\infty}(\Omega)} \leq M$ for $\epsilon>0$ small enough. Now, since $u_{0} \in W^{1, p(x)}(\Omega)$ is a $C^{1}$ local minimizer, $u_{0}$ is a weak solution to $\left(P_{\lambda}\right)$; that is, satisfies ess $\inf _{K} u_{0}>0$ over every compact set $K \subset \Omega$ and

$$
\begin{gathered}
\int_{\Omega}\left|\nabla u_{0}\right|^{p(x)-2} \nabla u_{0} \nabla \phi d x+\int_{\Omega}\left|u_{0}\right|^{p(x)-2} \phi d x \\
=\int_{\Omega} \frac{1}{u_{0}^{\delta(x)}} \phi d x-\int_{\Omega} f\left(x, u_{0}\right) \phi d x \\
-\lambda \int_{\partial \Omega}\left|u_{0}\right|^{q(x)-1} u_{0} \phi d x,
\end{gathered}
$$

for all $\phi \in C_{c}^{\infty}(\Omega)$. Therefore, for every function $w \in$ $W^{1, p(x)}(\Omega), u_{0}$ satisfies

$$
\begin{aligned}
\int_{\Omega} & \left|\nabla u_{0}\right|^{p(x)-2} \nabla u_{0} \nabla w d x+\int_{\Omega}\left|u_{0}\right|^{p(x)-2} w d x \\
= & \int_{\Omega} \frac{1}{u_{0}^{\delta(x)}} w d x-\int_{\Omega} f\left(x, u_{0}\right) w d x \\
& -\lambda \int_{\partial \Omega}\left|u_{0}\right|^{q(x)-1} u_{0} w d x .
\end{aligned}
$$

Similarly,

$$
\begin{aligned}
\int_{\Omega} & \left|\nabla u_{\epsilon}\right|^{p(x)-2} \nabla u_{\epsilon} \nabla w d x+\int_{\Omega}\left|u_{\epsilon}\right|^{p(x)-2} w d x \\
= & \int_{\Omega} \frac{1}{u_{\epsilon}^{\delta(x)}} w d x-\int_{\Omega} f\left(x, u_{\epsilon}\right) w d x \\
& -\lambda \int_{\partial \Omega}\left|u_{\epsilon}\right|^{q(x)-1} u_{\epsilon} w d x .
\end{aligned}
$$

Now, substracting the above relations with $w=\left(u_{\epsilon}-u_{0}\right) \mid u_{\epsilon}-$ $\left.u_{0}\right|^{\beta-1}$, with $\beta>1$, as a test function in $\left(P_{\epsilon}\right)$, integrate by parts and use the fact that $u \mapsto-\Delta_{p(x)} u+|u|^{p(x)-1} u$ is a monotone operator to obtain

$$
\begin{aligned}
& -\mu_{\varepsilon}\left[\int_{\Omega}\left|u_{\varepsilon}-u_{0}\right|^{\alpha(x)}\left|u_{\epsilon}-u_{0}\right|^{\beta-1}\left(u_{\varepsilon}-u_{0}\right) d x\right. \\
& \left.+\int_{\partial \Omega}\left|u_{\varepsilon}-u_{0}\right|^{p^{-}+\beta-1} d x\right] \leq \int_{\Omega}\left(\frac{1}{u_{\varepsilon}^{\delta(x)}}-\frac{1}{u_{0}^{\delta(x)}}\right)
\end{aligned}
$$




$$
\begin{aligned}
& \cdot\left|u_{\epsilon}-u_{0}\right|^{\beta-1}\left(u_{\epsilon}-u_{0}\right) d x \\
& +\int_{\Omega}\left(f\left(x, u_{\varepsilon}\right)-f\left(x, u_{0}\right)\right)\left|u_{\epsilon}-u_{0}\right|^{\beta-1} \\
& \cdot\left(u_{\epsilon}-u_{0}\right) d x+\lambda \int_{\partial \Omega}\left(\left|u_{\varepsilon}\right|^{q-1} u_{\varepsilon}\left|u_{0}\right|^{q-1} u_{0}\right) \\
& \cdot\left|u_{\epsilon}-u_{0}\right|^{\beta-1}\left(u_{\epsilon}-u_{0}\right) d x .
\end{aligned}
$$

Using the bounds of $u_{\epsilon}, u_{0}$ we get

$$
\begin{aligned}
& -\mu_{\varepsilon}\left[\int_{\Omega}\left|u_{\varepsilon}-u_{0}\right|^{\alpha(x)}\left|u_{\epsilon}-u_{0}\right|^{\beta-1}\left(u_{\varepsilon}-u_{0}\right) d x\right. \\
& \left.+\lambda \int_{\partial \Omega}\left|u_{\varepsilon}-u_{0}\right|^{p^{-}+\beta-1} d x\right] \leq C\left[\int_{\Omega}\left|u_{\epsilon}-u_{0}\right|^{\beta} d x\right. \\
& \left.\quad+\lambda \int_{\partial \Omega}\left|u_{\epsilon}-u_{0}\right|^{\beta} d x\right]
\end{aligned}
$$

where $C$ does not depend on $\beta$ and $\epsilon$. Now, using Hölder's inequality and the bounds of $u_{\epsilon}$ combined with Lemma 4 we obtain

$$
\begin{aligned}
\int_{\Omega}\left|u_{\varepsilon}\right|^{\beta} & \leq C\left\|u_{\varepsilon}\right\|_{L^{\alpha(x)+\beta-1}}^{\beta} \\
& \leq\left(\int_{\Omega}\left|u_{\varepsilon}\right|^{\alpha(x)+\beta-1}\right)^{\beta /\left(\alpha_{-}+\beta-1\right)} .
\end{aligned}
$$

Therefore

$$
\begin{aligned}
& -\mu_{\varepsilon}\left[\int_{\Omega}\left|u_{\varepsilon}-u_{0}\right|^{\alpha(x)}\left|u_{\epsilon}-u_{0}\right|^{\beta-1}\left(u_{\varepsilon}-u_{0}\right) d x\right. \\
& \left.+\lambda \int_{\partial \Omega}\left|u_{\varepsilon}-u_{0}\right|^{p^{-}+\beta-1} d x\right] \leq C(|\Omega|) \\
& \cdot\left[\int_{\Omega}\left|u_{\epsilon}-u_{0}\right|^{\alpha(x)+\beta-1} d x\right. \\
& \left.+\lambda \int_{\partial \Omega}\left|u_{\epsilon}-u_{0}\right|^{\alpha(x)+\beta-1} d x\right]^{\beta /\left(\alpha_{-}+\beta-1\right)} .
\end{aligned}
$$

Thus for any $\beta>1$

$$
\begin{aligned}
& -\mu_{\epsilon}\left[\left\|u_{\epsilon}-u_{0}\right\|_{L^{\alpha(x)+\beta-1}(\Omega)}^{\alpha_{-}-1}+\left\|u_{\epsilon}-u_{0}\right\|_{L^{\alpha(x)+\beta-1}(\partial \Omega)}^{\alpha_{-}-1}\right] \\
& \leq C(|\Omega|) .
\end{aligned}
$$

Passing to the limit in (79) $\beta \rightarrow+\infty$ we get

$$
\begin{aligned}
& \sup \left(-\mu_{\epsilon}\right)\left[\left\|u_{\epsilon}-u_{0}\right\|_{L^{\infty}(\Omega)}^{\alpha_{-}-1}+\left\|u_{\epsilon}-u_{0}\right\|_{L^{\infty}(\partial \Omega)}^{\alpha_{-}-1}\right] \\
& \quad \leq C(|\Omega|) .
\end{aligned}
$$

Then, using (80) combined with Proposition 15, the uniform $L^{\infty}$ bounds for $\left\{u_{\varepsilon}\right\}_{\varepsilon \in(0,1)}$ in $\Omega$ as well as $\partial \Omega$, we get that the right-hand side terms in $\left(P_{\varepsilon}\right)$ are uniformly bounded in $L^{\infty}(\Omega)$ and in $L^{\infty}(\partial \Omega)$ from which as in the first case we obtain that $u_{\epsilon}(0<\epsilon \leq 1)$ is bounded in $C^{1, \alpha}(\bar{\Omega})$ independently of $\epsilon$. Finally, using Ascoli-Arzela Theorem we find a sequence $\epsilon_{n} \rightarrow 0^{+}$such that

$$
u_{\epsilon_{n}} \rightarrow u_{0} \quad \text { in } C^{1}(\bar{\Omega})
$$

It follows that, for $\epsilon>0$ sufficiently small,

$$
E_{\lambda}\left(u_{\epsilon_{n}}\right)<E_{\lambda}\left(u_{0}\right)
$$

which contradicts the fact that $u_{0}$ is a local minimizer of $E_{\lambda}$ for the $C^{1}(\bar{\Omega}) \cap C_{0}(\bar{\Omega})$ topology. The proof of Lemma 14 is now completed.

\section{Existence of a Second Weak Solution for$$
0<\lambda<\Lambda
$$

In this section, we fix $\lambda \in(0, \Lambda)$ and let $0<\lambda_{1}<\lambda<$ $\lambda_{2}<\Lambda, u_{\lambda_{1}}$ and $u_{\lambda_{2}}$ be as in Section 3. Now, we are able to show the existence of a second solution using the generalized Mountain Pass Theorem. Since the functional is not $C^{1}$, we use the cut-off functional $\bar{E}_{\lambda}$ defined in (86). Define the cutoff functions

$$
\begin{aligned}
\bar{g}: \Omega \times \mathbb{R} \rightarrow \mathbb{R} \text { by } \\
\bar{g}(x, s)= \begin{cases}\frac{1}{s^{\delta(x)}}, & \text { if } s>u_{\lambda}(x), \\
\frac{1}{u(x)_{\lambda}^{\delta(x)}}, & \text { if } s \leq u_{\lambda}(x),\end{cases}
\end{aligned}
$$

$$
\begin{aligned}
\bar{f}: \Omega \times \mathbb{R} \rightarrow \mathbb{R} \text { by } \\
\bar{f}(x, s)= \begin{cases}f(x, s), & \text { if } s>u_{\lambda}(x), \\
f\left(x, u_{\lambda}(x)\right), & \text { if } s \leq u_{\lambda}(x),\end{cases}
\end{aligned}
$$

$$
\begin{aligned}
\bar{h}: \partial \Omega \times \mathbb{R} \rightarrow \mathbb{R} \text { by } \\
\bar{h}(x, s)= \begin{cases}\psi(x) s^{q(x)}, & \text { if } s>u_{\lambda}(x), \\
\psi(x) u_{\lambda}^{q(x)}(x), & \text { if } s \leq u_{\lambda}(x),\end{cases}
\end{aligned}
$$

and define now the corresponding functional $\bar{E}_{\lambda}: W^{1, p(x)}(\Omega)$ $\rightarrow \mathbb{R}$ given by

$$
\begin{aligned}
\bar{E}_{\lambda}(u)= & \int_{\Omega} \frac{|\nabla u|^{p(x)}}{p(x)}+\int_{\Omega} \frac{|u|^{p(x)}}{p(x)}-\int_{\Omega} \bar{G}(x, u) d x \\
& -\int_{\Omega} \bar{F}(x, u) d x-\lambda \int_{\partial \Omega} \bar{H}(x, u) d x,
\end{aligned}
$$

where $\bar{G}(x, u)=\int_{0}^{u} \bar{g}(x, t) d t, \bar{F}(x, u)=\int_{0}^{u} \bar{f}(x, t) d t$, and $\bar{H}(x, u)=\int_{0}^{u} \bar{h}(x, t) d t$. Firstly, we prove the following lemma on compactness of Palais-Smale sequences.

Lemma 17. The functional $\bar{E}_{\lambda}$ satisfies the Palais-Smale condition. 
Proof. Let $\left\{u_{n}\right\}$ be a (PS) sequence; namely, $\bar{E}_{\lambda}\left(u_{n}\right)$ is bounded and $\bar{E}_{\lambda}^{\prime}\left(u_{n}\right) \rightarrow 0$ when $n \rightarrow \infty$. Then,

$$
\begin{aligned}
c & \geq E_{\lambda}\left(u_{n}\right)-\frac{1}{\theta}\left\langle E_{\lambda}^{\prime}\left(u_{n}\right), u_{n}\right\rangle+\frac{1}{\theta}\left\langle E_{\lambda}^{\prime}\left(u_{n}\right), u_{n}\right\rangle \\
& \geq \int_{\Omega} \frac{\left|\nabla u_{n}\right|^{p(x)}}{p(x)}+\int_{\Omega} \frac{\left|u_{n}\right|^{p(x)}}{p(x)}-\int_{\Omega} \bar{G}\left(x, u_{n}\right) \\
& -\int_{\Omega} \bar{F}\left(x, u_{n}\right)-\frac{1}{\theta}\left[\int_{\Omega} \frac{\left|\nabla u_{n}\right|^{p(x)}}{p(x)}+\int_{\Omega} \frac{\left|u_{n}\right|^{p(x)}}{p(x)}\right. \\
& \left.-\int_{\Omega} \bar{G}\left(x, u_{n}\right)-\int_{\Omega} \bar{F}\left(x, u_{n}\right)-\lambda \int_{\partial \Omega} \bar{H}\left(x, u_{n}\right)\right] \\
& +\frac{1}{\theta}\left\langle E_{\lambda}^{\prime}\left(u_{n}\right), u_{n}\right\rangle .
\end{aligned}
$$

Now, we estimate the boundary term from above as follows:

$$
\begin{aligned}
\int_{\partial \Omega} \bar{H}\left(x, u_{n}\right)= & \int_{\partial \Omega \cap\left\{u_{n} \leq 1\right\}} \bar{H}\left(x, u_{n}\right) \\
& +\int_{\partial \Omega \cap\left\{u_{n}>1\right\}} \bar{H}\left(x, u_{n}\right) \\
\leq & K_{1}+\int_{\partial \Omega \cap\left\{u_{n} \leq 1\right\}} \frac{\left|u_{n}\right|^{q(x)+1}}{q(x)+1} \\
\leq & K_{1}+\frac{1}{q_{-}+1} \int_{\partial \Omega}\left|u_{n}\right|^{q^{+}+1} \\
\leq & K_{1}+K_{2}\left(\int_{\Omega}\left|\nabla u_{n}\right|^{p^{-}}\right)^{\left(q^{+}+1\right) / p^{-}}
\end{aligned}
$$

Hence, taking (88) in (87) and using (H2) combined with (7) we get

$$
\begin{aligned}
\geq & \left(\frac{1}{p^{+}}-\frac{1}{\theta}\right) \int_{\Omega}\left(\left|\nabla u_{n}\right|^{p(x)}+\left|u_{n}\right|^{p(x)}\right) \\
& -K_{1}\left(\int_{\Omega}\left|\nabla u_{n}\right|^{p_{-}}\right)^{\left(q_{+}+1\right) / p_{-}}+\frac{1}{\theta}\left\langle\bar{E}_{\lambda}^{\prime}\left(u_{n}\right), u_{n}\right\rangle \\
& -K_{2} \\
\geq & \left(\frac{1}{p^{+}}-\frac{1}{\theta}\right) \int_{\Omega}\left(\left|\nabla u_{n}\right|^{p(x)}+\left|u_{n}\right|^{p(x)}\right) \\
& -K_{1}\left(\int_{\Omega}\left|\nabla u_{n}\right|^{p_{-}}\right)^{\left(q_{+}+1\right) / p_{-}} \\
& -\frac{1}{\theta}\left\|\bar{E}_{\lambda}^{\prime}\left(u_{n}\right)\right\|\left\|u_{n}\right\|_{W^{1, p(x)}(\Omega)}-K_{2} .
\end{aligned}
$$

Now, using Lemma 4 and the fact that $L^{p(x)} \subset L^{p^{-}}(\Omega)$, we get

$$
\begin{aligned}
\left\|\nabla u_{n}\right\|_{L^{p(x)}}^{q^{+}+1} \geq & \left(\frac{1}{p^{+}}-\frac{1}{\theta}\right) \int_{\Omega}\left(\left|\nabla u_{n}\right|^{p(x)}+\left|u_{n}\right|^{p(x)}\right) \\
& -\frac{1}{\theta}\left\|\bar{E}_{\lambda}^{\prime}\left(u_{n}\right)\right\|\left\|u_{n}\right\|_{W^{1, p(x)}(\Omega)}-K_{2}
\end{aligned}
$$

$$
\begin{aligned}
& \geq\left(\frac{1}{p^{+}}-\frac{1}{\theta}\right)\left(\left\|\nabla u_{n}\right\|_{L^{p(x)}}^{p^{-}}+\left\|u_{n}\right\|_{L^{p(x)}}^{p^{-}}\right) \\
& -\frac{1}{\theta}\left(\left\|\nabla u_{n}\right\|_{L^{p(x)}}+\left\|u_{n}\right\|_{L^{p(x)}}\right)-K_{2} .
\end{aligned}
$$

Hence, $\left\|u_{n}\right\|_{W^{1, p(x)}(\Omega)}$ is bounded. Without loss of generality, we assume that there exists a subsequence of $\left\{u_{n}\right\}$ such that $u_{n} \rightarrow u_{0}$. Therefore, using Theorems 5 and 6 we get

$$
\begin{aligned}
& \lim _{n \rightarrow \infty} \int_{\Omega} \bar{g}\left(x, u_{n}\right)\left(u_{n}-u_{0}\right) \longrightarrow 0 \quad \text { as } n \longrightarrow \infty \\
& \lim _{n \rightarrow \infty} \int_{\Omega} \bar{f}\left(x, u_{n}\right)\left(u_{n}-u_{0}\right) \longrightarrow 0 \quad \text { as } n \longrightarrow \infty \\
& \lim _{n \rightarrow \infty} \int_{\partial \Omega} \bar{h}\left(x, u_{n}\right)\left(u_{n}-u_{0}\right) \longrightarrow 0 \quad \text { as } n \longrightarrow \infty
\end{aligned}
$$

Observe that

$$
\begin{aligned}
& \left\langle\bar{E}_{\lambda}\left(u_{n}\right), u_{n}-u_{0}\right\rangle=\int_{\Omega}\left(\left|\nabla u_{n}\right|^{p(x)-2} u_{n} \nabla\left(u_{n}-u_{0}\right)\right. \\
& \left.+\left|u_{n}\right|^{p(x)-2} u_{n}\left(u_{n}-u_{0}\right)\right)-\int_{\Omega} \bar{g}\left(x, u_{n}\right)\left(u_{n}-u_{0}\right) \\
& \quad-\int_{\Omega} \bar{f}\left(x, u_{n}\right)-\int_{\partial \Omega} \bar{h}\left(x, u_{n}\right)\left(u_{n}-u_{0}\right) .
\end{aligned}
$$

We already know that

$$
\left\langle\bar{E}_{\lambda}\left(u_{n}\right), u_{n}-u_{0}\right\rangle \longrightarrow 0 \text { as } n \longrightarrow \infty .
$$

Using (91), we obtain

$$
\begin{aligned}
& \int_{\Omega}\left(\left|\nabla u_{n}\right|^{p(x)-2} \nabla u_{n} \nabla\left(u_{n}-u_{0}\right)\right. \\
& \left.\quad+\left|u_{n}\right|^{p(x)-2} u_{n}\left(u_{n}-u_{0}\right)\right) \longrightarrow 0 \text { as } n \longrightarrow \infty
\end{aligned}
$$

This together with the convergence of $u_{n} \rightarrow u_{0}$ in $W^{1, p(x)}(\Omega)$ implies that $u_{n} \rightarrow u_{0}$ strongly in $W^{1, p(x)}(\Omega)$; that is, $\bar{E}_{\lambda}$ satisfies the (PS) condition. The proof of Lemma 17 is now completed.

Proof of Theorem 3. Firstly, note that $\bar{E}_{\lambda}(u) \geq \bar{E}_{\lambda}\left(u_{\lambda}\right)$ for any solution $u$ of (50). Hence, as in Section 3 we can conclude that $u_{\lambda}$ is a local minimum for $\bar{E}_{\lambda}$ in $W^{1, p(x)}(\Omega)$. By the strong comparison principle and Hopf lemma, we can conclude that any critical point of $\bar{E}_{\lambda}$ is also a critical point of $E_{\lambda}$ and hence $u$ also solves $\left(P_{\lambda}\right)$. It is easy to see that $u_{\lambda_{1}}$ and $u_{\lambda_{2}}$ are a subsolution and a supersolution to the problem associated with the functional energy $\bar{E}_{\lambda}$. Therefore, using the approach as in Theorem 2, we prove that this problem has a solution $v_{\lambda} \in\left[u_{\lambda_{1}}, u_{\lambda_{2}}\right] \cap C^{1}(\bar{\Omega})$ such that $v_{\lambda}$ is a local minimizer of $\bar{E}_{\lambda}$ in the $C^{1}$ topology. Now, by the comparison principle we can see that $v_{\lambda} \geq u_{\lambda}$ and also $v_{\lambda}$ solves $\left(P_{\lambda}\right)$. If $v_{\lambda} \not \equiv u_{\lambda}$ the conclusion of Theorem 3 holds. That is, we can assume $v_{\lambda} \equiv u_{\lambda}$ and $u_{\lambda}$ is a strict local minimum of $\bar{E}_{\lambda}$ in 
the $W^{1, p(x)}(\Omega)$ topology. Then, from Lemma A.4, the functional $\bar{E}_{\lambda} \in C^{1}\left(W^{1, p(x)}(\Omega), \mathbb{R}\right)$ and note that $\bar{E}_{\lambda}(t \varphi) \rightarrow-\infty$ as $t \rightarrow \infty$. Thus, we can apply Lemma 17 combined with the Mountain Pass Theorem to conclude that problem $\left(P_{\lambda}\right)$ has a solution $v_{\lambda}$ such that $v_{\lambda} \neq u_{\lambda}$. Therefore the proof of Theorem 3 is now completed.

\section{Appendix}

We start with an important technical tool which enables us to estimate the singularity in the Gâteaux derivative of the energy functional $E_{\lambda}: W^{1, p(x)}(\Omega) \rightarrow \mathbb{R}$ defined in (2).

Lemma A.1. Let $0<\delta<1$. Then there exists a constant $C_{\delta}>$ 0 such that the inequality

$$
\int_{0}^{1}|\mathbf{a}+s \mathbf{b}|^{-\delta} d s \leq C_{\delta}\left(\max _{0 \leq s \leq 1}|\mathbf{a}+s \mathbf{b}|\right)^{-\delta}
$$

holds true for all $\mathbf{a}, \mathbf{b} \in \mathbb{R}^{N}$ with $|\mathbf{a}|+|\mathbf{b}|>0$.

An elementary proof of this lemma can be found in Takáč [25, Lemma A.1, p. 233]. We continue by showing the Gâteaux differentiability of the energy functional $E_{\lambda}$ at a point $u \in$ $W^{1, p(x)}(\Omega)$ satisfying $u \geq C_{1}[\operatorname{dist}(x)]^{\theta_{1}}$ in $\Omega$ with $\theta_{1}=$ $\max _{\operatorname{dist}(x) \leq \sigma}(p(x) /(p(x)-1+\delta(x))$ ) (for details see Theorem 4.2 in [16]).

Lemma A.2. Let assumptions (H0)-(H5) be satisfied. Assume that $u, v \in W^{1, p(x)}(\Omega)$ and $u$ satisfies $u \geq$ $C_{1}[\operatorname{dist}(x)]^{\theta_{1}}$ in $\Omega$. Then we have

$$
\begin{aligned}
\lim _{t \rightarrow 0} & \frac{1}{t}\left(E_{\lambda}(u+t v)-E_{\lambda}(u)\right) \\
= & \int_{\Omega}|\nabla u|^{p(x)-2} \nabla u \cdot \nabla v d x+\int_{\Omega}|u|^{p(x)-2} u v d x \\
& -\int_{\Omega} \frac{1}{u^{\delta(x)}} v d x-\int_{\Omega} f(x, u) v d x \\
& -\lambda \int_{\partial \Omega}|u|^{q(x)} v d x .
\end{aligned}
$$

Proof. We show the result only for the singular term $\int_{\Omega}\left(v / u^{\delta(x)}\right) d x$; the other two terms are treated in a standard way. So let

$$
H(u)=\int_{\Omega} \frac{1}{u^{\delta(x)}} d x, \quad \text { for } u \in W^{1, p(x)}(\Omega) .
$$

For $\xi \in \mathbb{R} \backslash\{0\}$ we define

$$
z(\xi)=\frac{d}{d \xi} H\left(\xi^{+}\right)= \begin{cases}\frac{1}{u^{\delta(x)}}, & \text { if } \xi>0 \\ 0, & \text { if } \xi<0 .\end{cases}
$$

Consequently,

$$
\begin{aligned}
& \frac{1}{t}(H(u+t v)-H(u)) \\
& \quad=\int_{\Omega}\left(\int_{0}^{1} z(u+s t v) d s\right) v d x .
\end{aligned}
$$

Notice that for almost every $x \in \Omega$ we have $u(x)>0$ and

$$
\begin{aligned}
& \int_{0}^{1} z(u(x)+s t v(x)) d s \longrightarrow z(u(x))= \frac{1}{u^{\delta(x)}}, \\
& \text { as } t \longrightarrow 0 .
\end{aligned}
$$

Moreover, the integral on the left-hand side (with nonnegative integrand) is dominated by

$$
\begin{aligned}
& \int_{0}^{1} z(u(x)+s t v(x)) d s \\
& \quad \leq C \int_{0}^{1}|u(x)+s t v(x)|^{-\delta(x)} d s \\
& \quad \leq C_{\delta^{-}} \int_{0}^{1}|u(x)+s t v(x)|^{-\delta^{+}} d s \\
& \quad \leq C_{\delta^{-}}\left(\max _{0 \leq s \leq 1}|u(x)+s t v(x)|\right)^{-\delta^{+}} \leq C_{\delta^{-}} u^{-\delta^{+}} \\
& \quad \leq C_{\delta^{-}}\left(C_{1}[d(x)]^{\theta_{1}}\right)^{-\delta^{+}}=C_{\delta^{-}, C_{1}} d(x)^{-\delta^{+} \theta_{1}}
\end{aligned}
$$

with constants $C_{\delta^{-}, C_{1}}>0$ independent of $x \in \Omega$. Here, we have used the estimate (A.1) from Lemma A.1 above. Finally, we have $v d(x)^{-\delta^{+} \theta_{1}} \in L^{1}(\Omega)$, by $v \in W^{1, p(x)}(\Omega)$ and Hardy's inequality. Hence, we are allowed to invoke the Lebesgue dominated convergence theorem in (A.5) from which the lemma follows by letting $t \rightarrow 0$.

Corollary A.3. Let assumptions (H0)-(H5) be satisfied. Then the energy functional $E_{\lambda}: W^{1, p(x)}(\Omega) \rightarrow \mathbb{R}$ is Gâteaux differentiable at every point $u \in W^{1, p(x)}(\Omega)$ that satisfies $u \geq$ $C_{1}[d(x)]^{\theta_{1}}$ in $\Omega$. Its Gâteaux derivative $E_{\lambda}^{\prime}(u)$ at $u$ is given by

$$
\begin{aligned}
\left\langle E_{\lambda}^{\prime}(u), v\right\rangle= & \int_{\Omega}|\nabla u|^{p(x)-2} \nabla u \cdot \nabla v d x \\
& +\int_{\Omega}|u|^{p(x)-2} u v d x-\int_{\Omega} u^{-\delta(x)} v d x \\
& -\int_{\Omega} f(x, u) v d x-\lambda \int_{\partial \Omega}|u|^{q(x)} v d x
\end{aligned}
$$

for $v \in W^{1, p(x)}(\Omega)$.

We continue by proving the $C^{1}$-differentiability of the cutoff energy functional $\bar{E}_{\lambda}$ defined below.

Lemma A.4. Let assumptions (H0)-(H5) be satisfied, and $w \in W^{1, p(x)}(\Omega)$ such that $w \geq C_{1}[d(x)]^{\theta_{1}}$ in $\Omega$. Setting for $x \in \Omega$

$$
\begin{aligned}
& \bar{h}(x, s) \\
& = \begin{cases}s^{-\delta(x)}+f(x, s)+\lambda|s|^{q(x)}, & \text { if } s \geq w(x), \\
w^{-\delta(x)}(x)+f(x, w(x))+\lambda|w(x)|^{q(x)}, & \text { if } s<w(x),\end{cases}
\end{aligned}
$$




$$
\begin{array}{r}
\bar{H}(x, s)=\int_{0}^{s} h(x, t) d t \text { and for } u \in W^{1, p(x)}(\Omega) \\
\bar{E}_{\lambda}(u)=\int_{\Omega} \frac{|\nabla u|^{p(x)}}{p(x)} d x-\int_{\Omega} \bar{H}(x, u) d x,
\end{array}
$$

we have that $\bar{E}_{\lambda}$ belongs to $C^{1}\left(W^{1, p(x)}(\Omega), \mathbb{R}\right)$.

Proof. As in Lemma A.2, we concentrate on the singular term, the others being standard. Let

$$
g(x, s)= \begin{cases}s^{-\delta(x)}, & \text { if } s \geq w(x), \\ w^{-\delta(x)}(x), & \text { if } s<w(x),\end{cases}
$$

$G(x, s)=\int_{0}^{s} g(x, t) d t$, and $S(u)=\int_{\Omega} G(x, u) d x$. Proceeding as in Lemma A.2, we obtain that, for all $u \in W_{0}^{1, p(x)}(\Omega), S(u)$ has a Gâteaux derivative $S^{\prime}(u)$ given by

$$
\left\langle S^{\prime}(u), v\right\rangle=\int_{\Omega}(\max \{u(x), w(x)\})^{-\delta(x)} v(x) d x .
$$

Let $u_{k} \in W_{0}^{1, p(x)}(\Omega), u_{k} \rightarrow u_{0}$. Then

$$
\begin{aligned}
& \left|\left\langle S^{\prime}\left(u_{k}\right)-S^{\prime}\left(u_{0}\right), v\right\rangle\right| \\
& \quad=\mid \int_{\Omega}\left(\left(\max \left\{u_{k}(x), w(x)\right\}\right)^{-\delta(x)} v(x)\right. \\
& \left.\quad-\left(\max \left\{u_{0}(x), w(x)\right\}\right)^{-\delta(x)} v(x)\right) d x \mid \\
& \quad \leq 2 C \int_{\Omega} w^{-\delta(x)}|v| d x \leq 2 C \int_{\Omega} w^{-\delta^{+}}|v| d x \\
& \quad \leq 2 C C_{1}^{-\delta^{+}} \int_{\Omega}[d(x)]^{-\delta^{+} \theta_{1}}|v| d x
\end{aligned}
$$

for all $v \in W^{1, p(x)}(\Omega)$. Again, as in Lemma A.2, we use Hardy's inequality to deduce that $[d(x)]^{-\delta^{+} \theta_{1}} v \in L^{1}(\Omega)$, so that by Lebesgue's dominated convergence theorem we conclude that the Gâteaux derivative of $S$ is continuous which implies that $S \in C^{1}\left(W^{1, p(x)}(\Omega), \mathbb{R}\right)$.

\section{Competing Interests}

The authors declare that there is no conflict of interests regarding the publication of this article.

\section{Acknowledgments}

This work is supported by the Research Center, Scientific Research Deanship, University of Dammam, KSA, under Award no. 2015078.

\section{References}

[1] E. Acerbi and G. Mingione, "Regularity results for a class of functionals with non-standard growth," Archive for Rational Mechanics and Analysis, vol. 156, no. 2, pp. 121-140, 2001.
[2] L. Diening, Theorical and numerical results for electrorheological fluids [Ph.D. thesis], University of Freiburg, Freiburg im Breisgau, Germany, 2002.

[3] E. A. Abreu, J. M. do O, and E. S. Medeiros, "Multiplicity of positive solutions for a class of quasilinear nonhomogeneous Neumann problems," Nonlinear Analysis: Theory, Methods \& Applications, vol. 60, no. 8, pp. 1443-1471, 2005.

[4] Y. Deng and L. Jin, "Multiple positive solutions for a quasilinear nonhomogeneous Neumann problems with critical Hardy exponents," Nonlinear Analysis. Theory, Methods \& Applications. An International Multidisciplinary Journal. Series A: Theory and Methods, vol. 67, no. 12, pp. 3261-3275, 2007.

[5] J. Garcia-Azorero, I. Peral, and J. D. Rossi, "A convex-concave problem with a nonlinear boundary condition," Journal of Differential Equations, vol. 198, no. 1, pp. 91-128, 2004.

[6] K. Saoudi, "Multiple results for a singular elliptic equations in $\mathbb{R}^{2}$ with a sublinear Neumann boundary condition," Complex Variables and Elliptic Equations, In press.

[7] X. Fan and S.-G. Deng, "Multiplicity of positive solutions for a class of inhomogeneous Neumann problems involving the $p(x)$-Laplacian," Nonlinear Differential Equations and Applications, vol. 16, no. 2, pp. 255-271, 2009.

[8] K. Sreenadh and S. Tiwari, "Global multiplicity results for $p(x)$ Laplacian equation with nonlinear Neumann boundary condition," Differential and Integral Equations. An International Journal for Theory \& Applications, vol. 26, no. 7-8, pp. 815-836, 2013.

[9] X. Fan and D. Zhao, "On the Spaces $L^{p(x)}(\Omega)$ and $W^{m, p(x)}(\Omega)$," Journal of Mathematical Analysis and Applications, vol. 263, no. 2, pp. 424-446, 2001.

[10] X. Fan, J. Shen, and D. Zhao, "Sobolev embedding theorems for spaces $W^{k, p(x)}(\Omega)$," Journal of Mathematical Analysis and Applications, vol. 262, no. 2, pp. 749-760, 2001.

[11] O. Kovăčik and J. Răkosnik, "On spaces $L^{p(x)}$ and $W^{k, p(x), "}$ Czechoslovak Mathematical Journal, vol. 41(116), no. 4, pp. 592618, 1991.

[12] X. Fan, "Boundary trace embedding theorems for variable exponent Sobolev spaces," Journal of Mathematical Analysis and Applications, vol. 339, no. 2, pp. 1395-1412, 2008.

[13] K. Saoudi and A. Ghanmi, "A multiplicity results for a singular equation involving the $p(x)$-Laplace operator," Complex Variables and Elliptic Equations, 2016.

[14] H. Brézis and E. Lieb, "A relation between pointwise convergence of functions and convergence of functionals," Proceedings of the American Mathematical Society, vol. 88, no. 3, pp. 486490, 1983.

[15] L. Boccardo and F. Murat, "Almost everywhere convergence of the gradients of solutions to elliptic and parabolic equations," Nonlinear Analysis. Theory, Methods \& Applications. An International Multidisciplinary Journal. Series A: Theory and Methods, vol. 19, no. 6, pp. 581-597, 1992.

[16] Q. Zhang, "Existence and asymptotic behavior of positive solutions to $p(x)$-Laplacian equations with singular nonlinearities," Journal of Inequalities and Applications, vol. 9, Article ID 19349, 2007.

[17] G. M. Lieberman, "The conormal derivative problem for elliptic equations of variational type," Journal of Differential Equations, vol. 49, no. 2, pp. 218-257, 1983.

[18] H. Brezis and L. Nirenberg, "Minima locaux relatifs à $C^{1}$ et $H^{1}$," Comptes Rendus de l'Académie des Sciences-Series I: Mathematics, vol. 317, pp. 465-472, 1993. 
[19] J. P. García Azorero, I. Peral Alonso, and J. J. Manfredi, "Sobolev versus Hölder local minimizers and global multiplicity for some quasilinear elliptic equations," Communications in Contemporary Mathematics, vol. 2, no. 3, pp. 385-404, 2000.

[20] F. Brock, L. Iturriaga, and P. Ubilla, "A multiplicity result for the p-Laplacian involving a parameter," Annales Henri Poincaré, vol. 9, no. 7, pp. 1371-1386, 2008.

[21] D. G. de Figueiredo, J.-P. Gossez, and P. Ubilla, "Local 'superlinearity' and 'sublinearity' for the p-Laplacian," Journal of Functional Analysis, vol. 257, no. 3, pp. 721-752, 2009.

[22] J. Giacomoni and K. Saoudi, " $W_{0}^{1, p}$ versus $C^{1}$ local minimizers for a singular and critical functional," Journal of Mathematical Analysis and Applications, vol. 363, no. 2, pp. 697-710, 2010.

[23] K. Saoudi, " $W_{0}^{1, p(x)}$ versus $C^{1}$ local minimizers for a functional with critical growth," Journal of Partial Differential Equations, vol. 27, no. 2, pp. 115-124, 2014.

[24] K. Saoudi and M. Kratou, "Existence of multiple solutions for a singular and quasilinear equation," Complex Variables and Elliptic Equations. An International Journal, vol. 60, no. 7, pp. 893-925, 2015.

[25] P. Takáč, "On the Fredholm alternative for the $p$-Laplacian at the first eigenvalue," Indiana University Mathematics Journal, vol. 51, no. 1, pp. 187-237, 2002. 


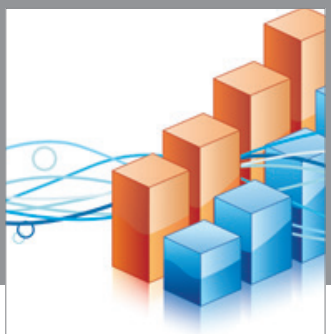

Advances in

Operations Research

vatem alat4

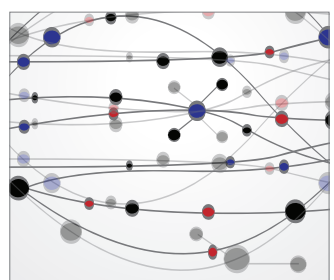

\section{The Scientific} World Journal
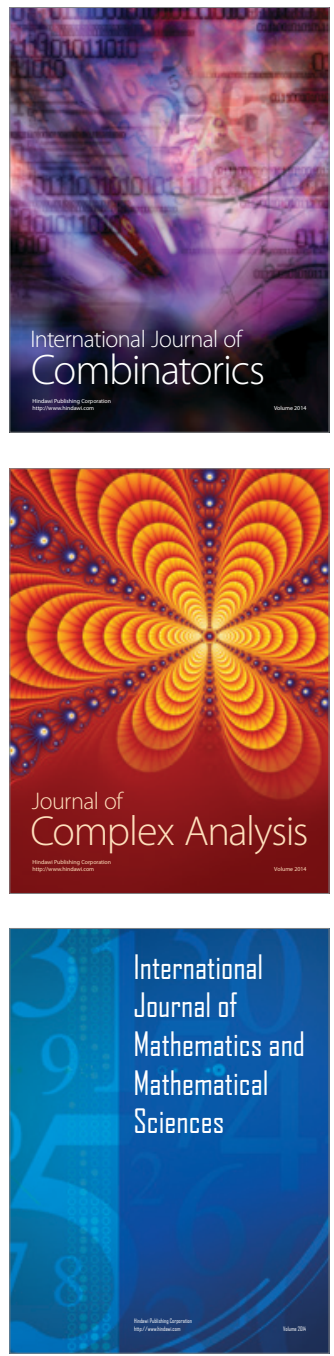
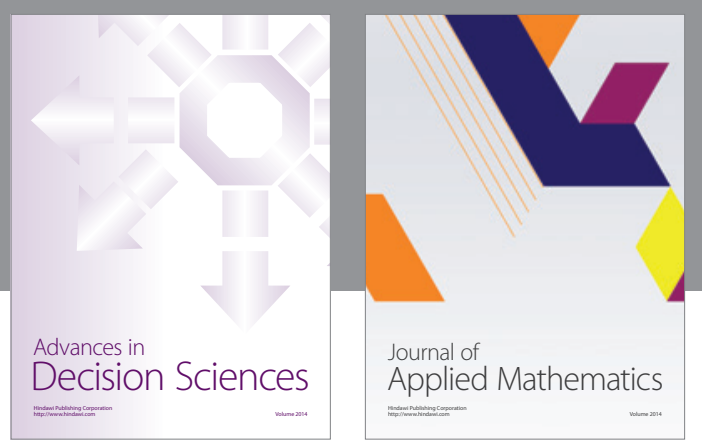

Algebra

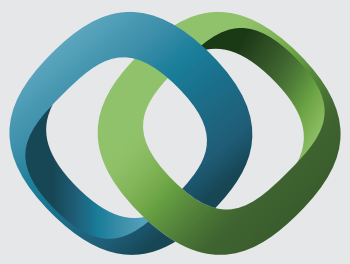

\section{Hindawi}

Submit your manuscripts at

http://www.hindawi.com
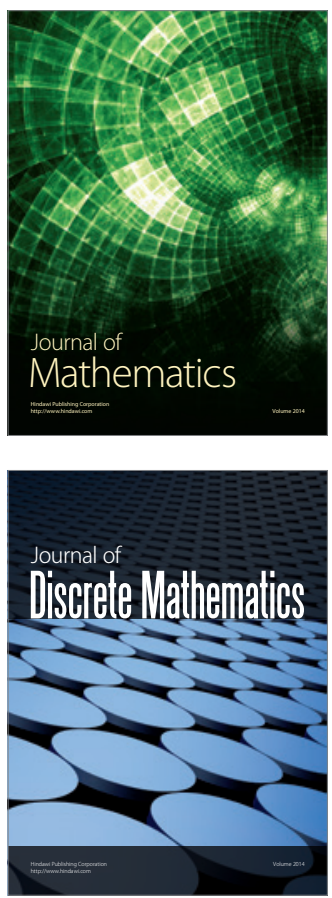

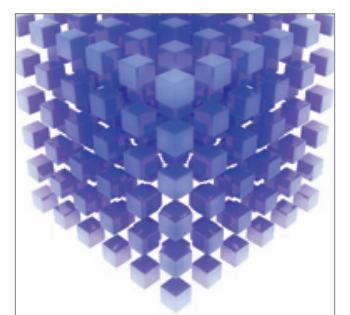

Mathematical Problems in Engineering
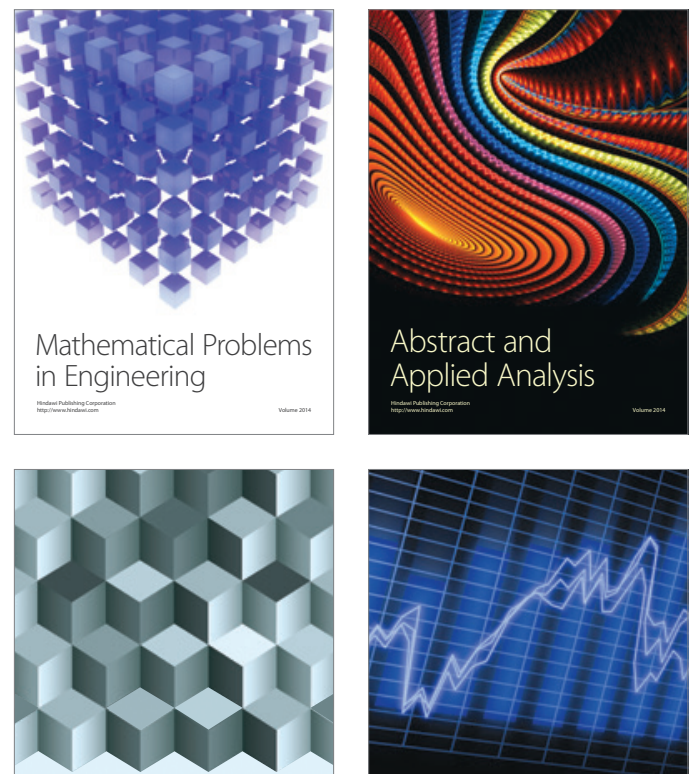

Journal of

Function Spaces

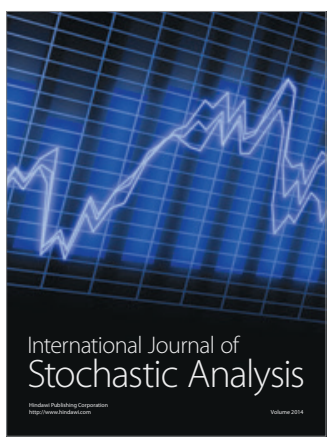

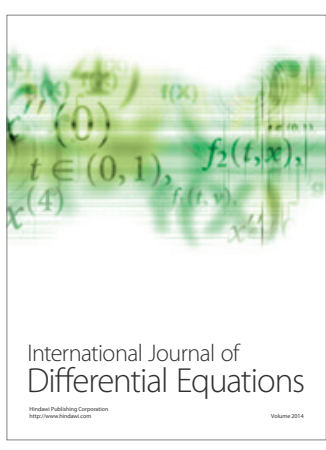
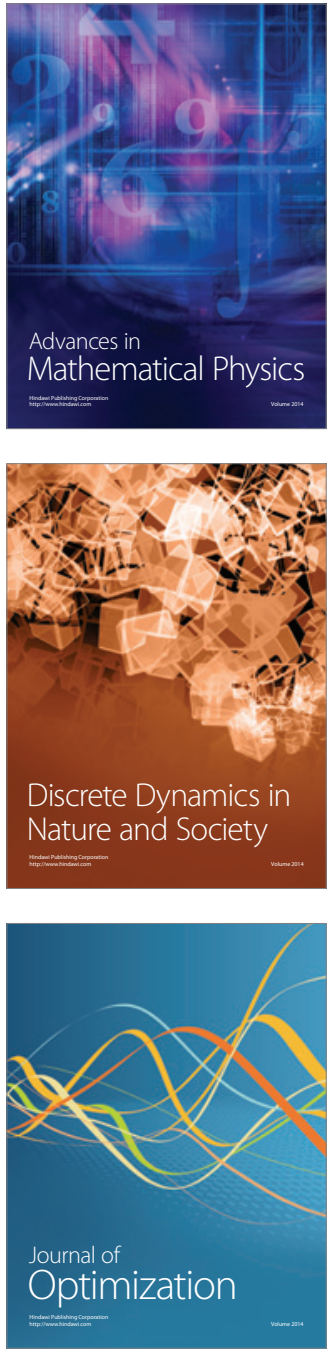